\title{
Competencias para la empleabilidad de los titulados en Pedagogía, Psicología y Psicopedagogía: un estudio comparativo entre empleadores y titulados Employability and competences of Pedagogy, Psychology, and Educational Psychology graduates: a comparative study of employers and graduates
}

Dra. Pilar PINEDA-HERRERO. Profesora Titular. Universidad Autónoma de Barcelona (pilar.pineda@uab.es). Anna CIRASO-CALI. Licenciada. Universidad Autónoma de Barcelona (annaciraso@gmail.com). Mary ARMIJOS-YAMBAY. Licenciada. Escuela Superior Politécnica del Litoral, Ecuador (marbarmi@espol.edu.ec).

\section{Resumen:}

La inserción laboral de los jóvenes universitarios se puede ver influenciada por varios factores, como la situación económica del país, la calidad de la formación universitaria 0 las exigencias del mercado laboral.

A pesar de que la economía española se ha ido recuperando, aún presenta cifras altas de desempleo juvenil, incluso entre los más formados. Por ello, es importante profundizar en el análisis de las competencias transversales que son demandadas y ofertadas en el mercado laboral, ya que de esto puede depender la inserción y satisfacción laboral de los graduados universitarios. En este artículo se analizan, a partir de los datos de la encuesta «Universitat i Treball a Catalunya» de AQU (2014a y 2014b) sobre los titulados en Pedagogía, Psicología y Psicopedagogía en las universidades catalanas y sus empleadores, las diferencias en las perspectivas de ambos colectivos respecto a las competencias necesarias para el mercado laboral.

Los resultados muestran que la visión de empleadores y titulados presenta algunas discrepancias. Por un lado, los titulados consideran más importantes algunas competencias como la toma de decisiones. Sin embargo, los empleadores dan mayor importancia a otras, como las que se relacionan con la informática y el conocimiento de idiomas, o la formación teórica y práctica impartida en la universidad. Ambos colectivos coinciden en posicionar el trabajo en equipo como la competencia más importante para el trabajo, confirmando los resultados obtenidos en otros estudios. Por otro lado, los empleadores están más satisfechos que los propios titulados con su nivel en los dominios competenciales de creatividad, informática e idiomas. La resolución de problemas, en cambio, emerge como un ámbito competencial que se podría potenciar en la formación universitaria.

Fecha de recepción de la versión definitiva de este artículo: 10-02-2018.

Cómo citar este artículo: Pineda-Herrero, P., Ciraso-Cali, A. y Armijos-Yambay, M. (2018). Competencias para la empleabilidad de los titulados en Pedagogía, Psicología y Psicopedagogía: un estudio comparativo entre empleadores y titulados | Employability and competences of Pedagogy, Psychology, and Educational Psychology graduates: a comparative study of employers and graduates. Revista Española de Pedagogía, 76 (270), 313-333. doi: https:// 
La contribución del estudio radica en aportar evidencias que puedan ayudar a alinear la formación universitaria con las expectativas de los empleadores, a la luz de la valoración retrospectiva de los recién titulados.

Descriptores: educación superior, competencias, ajuste formativo, empleadores, titulados, empleo juvenil, inserción laboral.

\section{Abstract:}

The labour market integration of recent graduates can be influenced by several factors, such as the economic situation of the country, the quality of their university education, and the demands of the labour market.

Although the Spanish economy has recently recovered somewhat, it still has high rates of youth unemployment, even among the best educated groups. Therefore, it is important to analyse in greater depth the

\section{Introducción y marco teórico}

La inserción laboral de los jóvenes es una cuestión que actualmente merece ser atendida a nivel social, político y académico. En España, la crisis económica ha dejado un legado que sobre todo afecta a los jóvenes titulados del país. Si bien es cierto que desde el 2015 el desempleo total ha disminuido, las cifras de desempleo juvenil actuales todavía son alarmantes. En the labour market this article uses data from the AQU's 'Universitat i Treball a Catalunya' survey (2014a and 2014b) of graduates in these subjects from Catalan universities and of their employers.

The results show discrepancies between employers' and graduates' views. On the one hand, graduates regard some competences as more relevant, such as decision-making, while employers give greater importance to others, such as those relating to information technology and language skills, as well as theoretical and practical training. However, both groups agree that teamwork is the most important competence for work, supporting results obtained in other studies. Furthermore, employers are more satisfied than the graduates with their level in the areas of creativity, computer science, and languages. Problem-solving, however, is an area of competence that could be enhanced in university education.

This study's contribution is to provide evidence based on the retrospective assessment of recent graduates that can help align university training with employers' expectations.

Keywords: higher education, competences, vocational adjustment, employers, graduates, youth employment, labour market integration.
Cataluña, una de las cinco comunidades autónomas con menor porcentaje de desempleo juvenil, la tasa de paro de los jóvenes alcanza el 30.46\% (datos del Instituto Nacional de Estadística-INE, Encuesta de Población Activa, 2017).

La situación laboral de los jóvenes con título universitario es especialmente preocupante, dado su rol estratégico para 
el futuro del país y la necesidad de rentabilizar la inversión, tanto privada como social, realizada en ellos. La encuesta de inserción laboral llevada a cabo en el 2014 por el INE apunta que los titulados universitarios graduados en el 2010 tenían una tasa de ocupación del $75.6 \%$ y una tasa de paro del 19.2\% en el 2014. Según las ramas de conocimiento y titulaciones, la tasa de paro de los graduados en ciencias sociales y jurídicas era del $20.4 \%$, por encima de la tasa en ciencias de la salud y arquitectura e ingeniería. De las tres titulaciones que se consideran en este estudio, la que mayor tasa de empleo presentó en el 2014 fue la Licenciatura de Psicopedagogía (83\%), seguida por la Licenciatura de Pedagogía (78\%) y finalmente la de Psicología (73.36\%). Sin embargo, se ha demostrado que los datos de inserción de los jóvenes profesionales de la educación (en concreto, egresados de las titulaciones de Pedagogía, Psicopedagogía y Magisterio) enmascaran problemas de sobrecualificación y empleo precario: según Pineda-Herrero, Agud-Morell y Ciraso-Calí (2016), solo el $10.9 \%$ de los jóvenes titulados en 2010 tiene un empleo satisfactorio en 2014 en el que desempeñan tareas adecuadas a su nivel formativo, con un salario anual mínimo que les permita la independencia económica.

El papel de la universidad en la formación de los jóvenes ha cambiado en la última década. La universidad actualmente tiene como uno de sus objetivos principales el de promover la empleabilidad (McCowan, 2015), papel de la universidad que también se ha visto impulsado por las políticas de empleabilidad de la Unión Europea, las cuales han originado debates sobre la relación entre educación universitaria y mercado laboral (Prokou, 2008). Pero, ¿realmente las universidades forman a sus estudiantes de manera que luego se puedan insertar adecuadamente en el mercado laboral?

En términos generales, la empleabilidad puede ser entendida como el conjunto de características, competencias y habilidades que permiten a una persona encontrar un trabajo con mayor facilidad (Álvarez y Miles, 2006). Según Fugate, Kinicki y Ashforth (2004) la empleabilidad es un constructo multidimensional formado por tres dimensiones: la identidad de la carrera, la adaptabilidad personal y el capital social y humano. En este caso, nos centraremos en la dimensión del capital humano, que hace referencia al proceso de desarrollo de conocimientos, aptitudes, habilidades y valores que mejorarán la satisfacción y desempeño laboral, a la vez que optimizarán el funcionamiento de la empresa (Marimuthu, Arokiasamy y Ismail, 2009). En esta línea, es necesario profundizar en el análisis de las competencias que son demandadas y ofertadas en el mercado laboral, ya que de esto puede depender la inserción y satisfacción laboral de los graduados universitarios (García-Aracil y Van der Velden, 2008).

Las competencias genéricas 0 transversales son comunes a la mayoría de las profesiones. Estas hacen referencia a los conocimientos básicos, la habilidad de analizar y sintetizar, organizar y planificar, la resolución de problemas, la toma de decisiones, la comunicación oral y escrita, los idiomas, las habilidades informáticas, el manejo de información, el pensamiento crítico, el trabajo en equipo, 
las habilidades interpersonales, el liderazgo, las habilidades para investigar, la autonomía, responsabilidad, motivación y autoestima (Carnevale, Gainer y Meltzer, 1989; Hernández-March, Martín del Peso y Leguey, 2009; Bernal, Delgado y Donoso, 2014). Autores como Wye y Lim (2009), Coleman (2011) y Cai (2013) recalcan la importancia de dominar idiomas para una buena inserción. Por otro lado, Carnevale y Smith (2013), y Stevens (2005), muestran que las habilidades de comunicación, como la comunicación oral, la escrita y la escucha activa, son habilidades muy valoradas en diversas ocupaciones. García y Pérez (2008) mencionan que, para alcanzar el nivel de productividad exigido por las empresas en esta era de la tecnología, son imprescindibles los conocimientos de informática e Internet. Así mismo, los empleadores valoran el trabajo en equipo (Weller, 2007), las habilidades interpersonales (Bridge, O’Neill y Cromie, 2003), la resolución de problemas y la toma de decisiones (Lantarón, 2014; Stasz, 1998).

En la literatura reciente se encuentran estudios, con enfoques tanto cuantitativos como cualitativos, sobre la empleabilidad y la inserción de los jóvenes en el mercado laboral. Martín-González, Ondé y Pérez-Esparrels (2015), haciendo uso del análisis factorial exploratorio y un modelo logit, examinaron el impacto de las competencias en la empleabilidad de los titulados de las universidades de Valencia, encontrando que la nota y la experiencia laboral e internacional durante los estudios universitarios favorecen la empleabilidad. Medir y Montolio (2015) estudiaron los factores asociados a la inserción laboral de los graduados catalanes en universidades públicas usando modelos de regresión lineal para realizar el análisis y evidenciaron un impacto positivo de la formación universitaria en la satisfacción en el empleo. Por otro lado, estudios como el de Jackling y De Lange (2009) usan métodos cualitativos, como las entrevistas, para analizar la convergencia 0 divergencia entre las habilidades adquiridas por los graduados en contabilidad y las expectativas de los empleadores. Entre los principales resultados está que los empleadores buscan jóvenes con habilidades genéricas (como trabajo en equipo, liderazgo, comunicación verbal y habilidades interpersonales), habilidades que los graduados consideran que no les fueron enseñadas durante sus estudios en la universidad; es decir, hallaron una divergencia. También se encuentran estudios con metodología mixta como el de Wickramasinghe y Perera (2010) que, mediante datos recogidos por encuestas, realizan pruebas paramétricas para analizar las diferencias en la opinión de los empleadores, profesores de universidad y graduados sobre las habilidades necesarias y adquiridas para el trabajo. Sus resultados indican que los tres grupos consideran como más importantes las habilidades de resolución de problemas, autoconfianza y trabajo en equipo.

Por otro lado, se encuentran estudios empíricos que analizan las competencias y la empleabilidad de los jóvenes desde la perspectiva de los graduados (como el de García, Sotelino y Crespo, 2014); o de los empleadores (Beaven y Wright, 2006, entre otros). Sin embargo, siguiendo a Freire y Teijeiro (2010), la aproximación que 
puede generar unos resultados más útiles es aquella que contrasta las perspectivas de ambos, como la que usaron Taylor (2005) en su evaluación sobre las expectativas de los empleadores y el ajuste con la percepción de los jóvenes, o Saunders y Zuzel (2015) en un estudio sobre la convergencia de perspectivas acerca de las cualidades personales, las habilidades transversales y el conocimiento específico.

El presente estudio se basará en el análisis de las percepciones de ambos agentes (jóvenes titulados en Pedagogía, Psicología y Psicopedagogía, y empleadores) acerca de sus competencias, la importancia que han tenido en su contratación y su satisfacción con la formación recibida; con la finalidad de evidenciar posibles desajustes y sugerir áreas de mejora de la formación universitaria para que los nuevos profesionales de la educación y la psicología sean más competentes en el mercado laboral.

\section{Método}

En el estudio nos planteamos los siguientes objetivos:

1) Analizar la percepción de las competencias de los titulados (importancia para el trabajo y satisfacción con la formación recibida) desde la perspectiva de los propios titulados.

2) Analizar la percepción de las competencias de los titulados (importancia para la actuación profesional y satisfacción con la formación), desde la perspectiva de los empleadores.

3) Determinar las diferencias, entre empleadores y titulados, en la valoración de las competencias (importan- cia para el trabajo y satisfacción con la formación recibida).

\section{Muestra}

Para este estudio, se ha hecho uso de la base de datos de la Agència per a la Qualitat del Sistema Universitari de Catalunya (AQU) sobre la inserción laboral de la población titulada de las universidades catalanas según la perspectiva de los titulados (AQU, 2014a) y los empleadores (AQU, 2014b).

La muestra de titulados del estudio incluye a graduados de todas las universidades públicas y privadas catalanas que se graduaron en el curso 2009-2010 y fueron encuestados en el 2014. Se configura con 17337 personas, que representan un porcentaje del $55 \%$ de la población de referencia (las personas tituladas en universidades catalanas en 2010), y un error muestral del $0.51 \%$. Para este artículo, se han tomado en cuenta a las personas con titulaciones en Pedagogía, Psicología y Psicopedagogía que, después de graduarse, tuvieron un trabajo ajustado con su formación. Tras aplicar estos criterios, la muestra se configuró por 328 casos de titulados: 207 con Licenciatura en Psicología, 75 en Pedagogía y 46 en Psicopedagogía.

En el caso de los empleadores, se consideraron a las empresas que emplearon a pedagogos, psicólogos y psicopedagogos. La muestra de empleadores se compone de 48 casos.

En la Tabla 1 se puede observar el resumen de las características de la muestra. 
TABLA 1. Descriptiva de titulados y empleadores.

\begin{tabular}{|c|c|c|c|}
\hline & \\
\hline & & Titulados & Empleadores \\
\hline $\begin{array}{l}\text { Situación la- } \\
\text { boral actual }\end{array}$ & $\begin{array}{l}\text { - Ocupados } \\
\text { - Parados }\end{array}$ & $\begin{array}{l}87 \% \\
10 \%\end{array}$ & \\
\hline $\begin{array}{l}\text { Ámbito de } \\
\text { ocupación }\end{array}$ & $\begin{array}{l}\text { - Empresas privadas } \\
\text { - Empresas públicas }\end{array}$ & $\begin{array}{l}74 \% \\
26 \%\end{array}$ & $\begin{array}{l}88 \% \\
12 \%\end{array}$ \\
\hline $\begin{array}{l}\text { Actividad } \\
\text { principal de } \\
\text { la empresa }\end{array}$ & $\begin{aligned} \text { - Sanidad y asistencia social } \\
\text { - Educación, investigación } \\
\text { y servicios culturales }\end{aligned}$ & $\begin{array}{l}44.2 \% \\
33.1 \%\end{array}$ & $\begin{array}{l}10 \% \\
44 \%\end{array}$ \\
\hline $\begin{array}{l}\text { Tamaño de } \\
\text { las empresas }\end{array}$ & $\begin{array}{l}\text { - Microempresa (<9 trabajadores) } \\
\text { - Pequeña empresa (10-50 trabajadores) } \\
\text { - Mediana empresa (51-250 trabajadores) } \\
\text { - Gran empresa (>250 trabajadores) }\end{array}$ & $\begin{array}{l}30 \% \\
27 \% \\
19 \% \\
22 \%\end{array}$ & $\begin{array}{l}21 \% \\
15 \% \\
46 \% \\
19 \%\end{array}$ \\
\hline \multicolumn{2}{|c|}{ Número de contratados recientemente titulados } & 1298 & \\
\hline
\end{tabular}

Fuente: Elaboración propia.

\section{Instrumentos y variables}

Para el estudio, se han utilizado los cuestionarios de la encuesta de la AQU a titulados (AQU, 2014a) y empleadores (AQU, 2014b). En el caso de los empleadores, para este artículo solo se ha hecho uso de los cuestionarios a empresas (omitiendo los cuestionarios para centros educativos y centros de salud, mayormente empleadores de titulados en Magisterio en el primer caso, y en Medicina y Enfermería en el segundo).

Para poder escoger los datos sobre las competencias a comparar entre empleadores y titulados, se escogieron preguntas específicas de cada cuestionario. En el caso del cuestionario administrado a los titulados, se seleccionó la sección «valoración de la formación recibida y su adecuación para el trabajo", en la que se pedía una valoración del 1 (muy bajo) a
7 (muy bueno) de 14 competencias respecto al nivel de formación recibido en la universidad y a su utilidad para el trabajo.

En cuanto al cuestionario para los empleadores, se trabajó con las respuestas del Bloque 3 llamado "competencias», en el que se valoraba, en una escala del 0 al 10, la importancia de un listado de 15 competencias para la actuación profesional y la satisfacción de los empleadores con la formación de los recién titulados en estas competencias.

La utilidad de las competencias para el trabajo (valorado en el cuestionario de los titulados) se comparó con el grado de importancia para el trabajo (valorado en el cuestionario de los empleadores). Asimismo, la valoración del nivel de formación recibida (del cuestionario de titulados) fue comparada con el grado de satisfacción 
con la formación de los titulados (del cuestionario de los empleadores). Se seleccionaron aquellas competencias que eran comunes en varios bloques y cuestionarios, 0 aquellas que podían ser comparadas directamente.

En el caso de los titulados, se creó una nueva variable con la media de las competencias de expresión oral y comunicación escrita para crear una sola variable denominada «comunicación». En el caso de los empleadores, se interpretó a la variable «creación de nuevas ideas y soluciones» como análoga a la variable «creatividad», en base a la acepción de creatividad proporcionada por Sefertzi (2000).

Finalmente, se trabajó con dos bloques (importancia para el trabajo y satisfacción con la formación) de 10 ítems, administrados tanto a titulados como a empleadores. Ambas escalas presentan buena fiabili$\operatorname{dad}(\alpha=.836$ y $\alpha=.886$ en la muestra de titulados, $\alpha=770$ y $\alpha=.886$ en la muestra de empleadores). Los 10 ítems de cada escala informan sobre las siguientes competencias, agrupados siguiendo la categorización empleada por la AQU (2014):

- Formación teórico-práctica: teoría y práctica.

- Competencias cognitivas: resolución de problemas, toma de decisiones y creatividad.

- Competencias instrumentales: comunicación, informática e idioma.

- Competencias interpersonales: trabajo en equipo y liderazgo.

Adicionalmente, se analizaron los ítems 14 y 15 del Bloque 2 del cuestionario para empleadores llamado «procesos de contratación» para profundizar en los factores que inciden en el proceso de contratación de los recientemente titulados. La pregunta 13, de tipo dicotómico, es sobre las dificultades de contratar personas adecuadas para el puesto de trabajo; en el caso de respuesta afirmativa, se planteaba un listado de razones para esta dificultad, pudiendo elegir más de una razón.

\section{Procedimiento}

Este estudio usa un diseño de investigación relacional-comparativo con un enfoque cuantitativo.

Después de unos primeros análisis descriptivos y exploratorios, para dar respuesta al primer y segundo objetivo, se analizó la valoración de la formación recibida y las competencias a nivel global y según el tamaño de la empresa, utilizando la prueba no paramétrica de suma de rangos U de Mann-Whitney; se aplicó esta prueba por el tipo de variables (ordinales) y por el no cumplimiento del supuesto de normalidad (supuesto evaluado con la prueba de Kolmogorov-Smirnov y exploración mediante gráficos $\mathrm{Q}-\mathrm{Q}$ normal y $\mathrm{Q}-\mathrm{Q}$ sin tendencias). Para el tercer objetivo, se estudió si convergía la perspectiva de los empleadores y titulados respecto a la importancia y satisfacción con el nivel de las competencias, también a través de la prueba U de Mann-Whitney. En todos los casos en que esta prueba reveló una diferencia significativa, se calculó la $r$ de Rosenthal (1991) como medida de tamaño del efecto. Al no disponer de estudios previos parecidos, se interpretó la magnitud de la $r$ siguiendo las indicaciones de $\mathrm{C}_{0}$ hen (1988). 
Debido a que la escala usada para medir las competencias es diferente en la encuesta de los titulados de la utilizada en la encuesta de los empleadores, se procedió a transformar las escalas para poder así homogeneizar los datos y comparar las competencias.

Para homogeneizar los datos, se recomiendan varios métodos, como el método de estrechamiento lineal, el método de referencia de distribución (De Jonge, Veenhooven y Arends, 2014), las regresiones para estimar la nueva escala (Colman, Norris y Preston, 1997) y la fórmula propuesta por Preston y Colman (2000), que es la que usamos en este estudio. La fórmula es la siguiente: (puntuación-1) / (número de categorías de respuesta-1)*100. Con el fin de que los resultados sean más fáciles de interpretar, se usó la fórmula siguiendo a Dawes (2008), multiplicando por 10 en vez de por 100. De esta forma, solo tuvimos que recategorizar la escala de los titulados, pues la de los empleadores es originalmente de 0 a 10 .

\section{Resultados}

A continuación, se presentan los resultados obtenidos, organizados según los objetivos del estudio.

\subsection{Análisis de la visión de las com- petencias desde la perspectiva de los titulados}

Para responder al primer objetivo del estudio (analizar la percepción de las competencias de los titulados desde la perspectiva de los propios titulados), en primer lugar, se realizó un análisis descriptivo de la valoración general respecto a la formación recibida y a las competencias desarrolladas.

En la Tabla 2 se observa que los titulados otorgan gran importancia a las competencias de trabajo en equipo, resolución de problemas, toma de decisiones y comunicación. La valoración que realizan de estas competencias respecto a la formación universitaria recibida muestra puntuaciones que se sitúan por encima de la mitad de la escala (del 0 al 10); aunque hay que considerar la asimetría negativa de todas las distribuciones, sobre todo la valoración del trabajo en equipo, lo que implica la existencia de puntuaciones negativas más desviadas de la media.

Los titulados manifiestan mayor satisfacción con la formación teórica recibida en la universidad, con el logro de las competencias de trabajo en equipo, comunicación, solución de problemas y toma de decisiones; aunque por lo general, la satisfacción media sobre la formación recibida no es elevada, destacando negativamente la formación en idiomas. También es destacable que las competencias en idiomas no se consideran, por parte de los titulados de la muestra, como muy relevantes para su trabajo. Sin embargo, es conveniente resaltar la dispersión de las respuestas, destacando sobre todo las mayores desviaciones típicas en las valoraciones de la creatividad, las competencias informáticas e idiomas. 
TABLA 2. Descripción de la valoración de las competencias (importancia para el trabajo y satisfacción con la formación recibida) por los titulados.

\begin{tabular}{|c|c|c|c|c|c|c|}
\hline & & Media & Mediana & $\begin{array}{c}\text { Desv. } \\
\text { Estándar }\end{array}$ & Simetría & Curtosis \\
\hline Formación y con & npetencias: in & portan & ia para el & rabajo & & \\
\hline Formación & Teoría & 5.737 & 6.670 & 2.461 & -0.397 & -0.212 \\
\hline teórico práctica & Práctica & 5.543 & 6.670 & 3.144 & -0.277 & -1.003 \\
\hline & $\begin{array}{l}\text { Solución de } \\
\text { problemas }\end{array}$ & 8.130 & 8.330 & 2.194 & -1.447 & 2.029 \\
\hline $\begin{array}{l}\text { Competencias } \\
\text { cognitivas }\end{array}$ & \begin{tabular}{|l} 
Toma de \\
decisiones
\end{tabular} & 7.884 & 8.330 & 2.310 & -1.282 & 1.353 \\
\hline & Creatividad & 6.885 & 7.500 & 2.812 & -0.914 & 0.081 \\
\hline & Comunicación & 7.642 & 8.330 & 2.175 & -1.092 & 0.816 \\
\hline $\begin{array}{l}\text { Competencias } \\
\text { instrumentales }\end{array}$ & Informática & 6.392 & 6.670 & 2.904 & -0.614 & -0.462 \\
\hline & Idioma & 4.969 & 5.000 & 3.500 & -0.069 & -1.298 \\
\hline Competencias & $\begin{array}{l}\text { Trabajo } \\
\text { en equipo }\end{array}$ & 8.226 & 8.330 & 2.064 & -1.189 & 0.979 \\
\hline & Liderazgo & 6.570 & 6.670 & 2.557 & -0.639 & -0.192 \\
\hline Formación y con & npetencias: se & tisfacci & in con la f & mación re & cibida & \\
\hline Formación & Teoría & 6.591 & 6.670 & 2.061 & -0.519 & 0.173 \\
\hline teórico práctica & Práctica & 5.081 & 5.000 & 2.848 & -0.069 & -0.811 \\
\hline & $\begin{array}{l}\text { Solución de } \\
\text { problemas }\end{array}$ & 5.843 & 6.670 & 2.503 & -0.459 & -0.450 \\
\hline $\begin{array}{l}\text { Competencias } \\
\text { cognitivas }\end{array}$ & $\begin{array}{l}\text { Toma de } \\
\text { decisiones }\end{array}$ & 5.571 & 5.000 & 2.632 & -0.220 & -0.771 \\
\hline & Creatividad & 4.700 & 5.000 & 2.888 & -0.008 & -0.978 \\
\hline & Comunicación & 5.889 & 5.835 & 2.425 & -0.343 & -0.488 \\
\hline $\begin{array}{l}\text { Competencias } \\
\text { instrumentales }\end{array}$ & Informática & 4.518 & 5.000 & 2.858 & 0.069 & -0.950 \\
\hline & Idioma & 2.434 & 1.670 & 2.684 & 0.866 & -0.248 \\
\hline Competencias & $\begin{array}{l}\text { Trabajo } \\
\text { en equipo }\end{array}$ & 7.276 & 8.330 & 2.309 & -0.698 & -0.067 \\
\hline & Liderazgo & 4.640 & 5.000 & 2.671 & -0.020 & -0.786 \\
\hline
\end{tabular}

Fuente: Elaboración propia.

Para profundizar en estos resultados, se han analizado las valoraciones de los titulados buscando eventuales diferencias según el tamaño de la empresa en la que trabajan.
El Gráfico 1 muestra la comparación de la valoración acerca de la utilidad de la formación recibida por parte de los titulados según el tamaño de las empresas 
donde trabajan (micro y pequeña empresa; mediana y gran empresa). La prueba U de Mann-Whitney indica que las competencias informáticas, idiomas y trabajo en equipo están consideradas como más importantes por los recién titulados que trabajan en empresas medianas y grandes (con rangos promedios de 176.47, 180.32 y 171.88 respectivamente), respecto a los que trabajan en micro y pequeña empresa (rangos promedios de 148.22, 145.47 y 151.51 respectivamente), $\mathrm{U}=10178.5$, $9667,10789.5$ con un valor de significación $p<.05$. El tamaño del efecto, en las tres competencias, se puede considerar pequeño, siguiendo a Rosenthal (1991) y Cohen (1988), con valores $r$ de .15, 0.19 y .12 respectivamente).

GRÁFICo 1. Valoración de la utilidad de la formación recibida y de las competencias para el trabajo (medianas), según las personas tituladas. Diferencias por tamaño de empresa donde trabajan.

En el bloque del nivel de satisfacción de la formación recibida, la prueba U indica que la satisfacción respecto a la formación en creatividad está mejor valorada por los titulados que trabajan en la mediana y gran empresa (con rango promedio de 172.62) en comparación con los que trabajan en micro y pequeña empresa (rango promedio de 150.97, $\mathrm{U}=10690$, $p=.036)$. El tamaño del efecto se considera pequeño $(r=, 12)$. Las demás competencias no presentan diferencias apreciables según el tamaño a un nivel de significación del .05 . 
6.2. Análisis de las competencias des- tiva de los empleadores), se presenta en la de la perspectiva de los empleadores Tabla 3 el análisis descriptivo de la valo-

Avanzando en el segundo objetivo del ración general por parte de los empleadoestudio (analizar la percepción de las com- res de la muestra acerca de la formación petencias de los titulados desde la perspec- realizada por los titulados contratados.

TABLA 3. Descripción de la valoración de las competencias

(importancia para el trabajo y satisfacción con la formación) por los empleadores.

\begin{tabular}{|c|c|c|c|c|c|c|}
\hline & & Media & Mediana & \begin{tabular}{|c|} 
Desv. \\
Estándar
\end{tabular} & Simetría & Curtosis \\
\hline \multicolumn{7}{|c|}{ Formación y competencias: importancia para el trabajo } \\
\hline \multirow{2}{*}{$\begin{array}{l}\text { Formación } \\
\text { teórico práctica }\end{array}$} & Teoría & 7.375 & 8.000 & 1.817 & -1.432 & 4.586 \\
\hline & Práctica & 7.750 & 8.000 & 2.119 & -1.253 & 2.448 \\
\hline \multirow{3}{*}{$\begin{array}{l}\text { Competencias } \\
\text { cognitivas }\end{array}$} & $\begin{array}{l}\text { Solución de } \\
\text { problemas }\end{array}$ & 8.128 & 8.000 & 1.752 & -0.912 & 0.385 \\
\hline & $\begin{array}{l}\text { Toma de } \\
\text { decisiones }\end{array}$ & 7.667 & 8.000 & 1.629 & -0.450 & -0.351 \\
\hline & \begin{tabular}{|l|} 
Creatividad \\
\end{tabular} & 8.043 & 8.000 & 1.517 & -0.465 & -0.417 \\
\hline \multirow{3}{*}{$\begin{array}{l}\text { Competencias } \\
\text { instrumentales }\end{array}$} & Comunicación & 8.354 & 8.500 & 1.537 & -1.071 & 1.762 \\
\hline & Informática & 8.021 & 8.000 & 1.657 & -0.738 & 0.026 \\
\hline & Idioma & 7.125 & 8.000 & 2.455 & -0.817 & 0.186 \\
\hline \multirow{2}{*}{$\begin{array}{l}\text { Competencias } \\
\text { interpersonales }\end{array}$} & $\begin{array}{l}\text { Trabajo } \\
\text { en equipo }\end{array}$ & 8.521 & 9.000 & 1.598 & -1.019 & 1.251 \\
\hline & Liderazgo & 6.674 & 7.000 & 2.066 & -0.520 & 0.546 \\
\hline \multicolumn{7}{|c|}{ Formación y competencias: nivel/satisfacción } \\
\hline \multirow{2}{*}{$\begin{array}{l}\text { Formación } \\
\text { teórico práctica }\end{array}$} & Teoría & 7.244 & 8.000 & 1.861 & -1.438 & 3.986 \\
\hline & Práctica & 5.511 & 6.000 & 2.212 & -0.319 & -0.012 \\
\hline \multirow{3}{*}{$\begin{array}{l}\text { Competencias } \\
\text { cognitivas }\end{array}$} & $\begin{array}{l}\text { Solución de } \\
\text { problemas }\end{array}$ & 6.273 & 6.000 & 1.744 & 0.221 & 0.317 \\
\hline & $\begin{array}{l}\text { Toma de } \\
\text { decisiones }\end{array}$ & 5.978 & 6.000 & 1.832 & 0.192 & 0.414 \\
\hline & Creatividad & 6.444 & 7.000 & 1.791 & -0.265 & 0.915 \\
\hline \multirow{3}{*}{$\begin{array}{l}\text { Competencias } \\
\text { instrumentales }\end{array}$} & Comunicación & 6.622 & 6.000 & 2.037 & -0.455 & 1.346 \\
\hline & Informática & 7.044 & 7.000 & 2.163 & -0.765 & 0.521 \\
\hline & Idioma & 6.068 & 6.500 & 2.204 & -0.241 & -0.413 \\
\hline \multirow{2}{*}{$\begin{array}{l}\text { Competencias } \\
\text { interpersonales }\end{array}$} & \begin{tabular}{|l} 
Trabajo \\
en equipo
\end{tabular} & 7.000 & 7.000 & 1.638 & -0.227 & 0.471 \\
\hline & Liderazgo & 5.273 & 6.000 & 2.316 & -0.042 & 0.087 \\
\hline
\end{tabular}

Fuente: Elaboración propia. 
Se puede observar que los empleadores otorgan importancia a las competencias de trabajo en equipo, resolución de problemas, comunicación y creatividad, siendo estas mismas competencias las mejor valoradas mediamente respecto al nivel competencial logrado por los titulados contratados. La competencia con la cual los empleadores están menos satisfechos es la de liderazgo (que es también la competencia a la que otorgan menor importancia para el trabajo); sin embargo, es necesario advertir nuevamente sobre la alta variabilidad de las respuestas.

Como en el apartado anterior, se han analizado estos resultados buscando eventuales diferencias entre la valoración de las competencias según el tamaño de la empresa (micro y pequeña empresa; mediana y gran empresa), utilizando la prueba U de Mann-Whitney. Como se observa en los Gráficos 2 y 3, la única diferencia estadísticamente significativa a $p<.05$ se halla en la valoración acerca de las competencias en idiomas: los empleadores de empresas medianas y grandes las valoran como más importantes (rango promedio de 27.89) que los empleadores de la micro y pequeña empresa (rango promedio de 18.32, $\mathrm{U}=158.5, p=.021)$. La magnitud de esta diferencia es moderada $(r=.33)$.

GRÁFICO 2. Valoración de la importancia de la formación recibida y de las competencias para el trabajo (medianas), según los empleadores. Diferencias por tamaño de empresa.

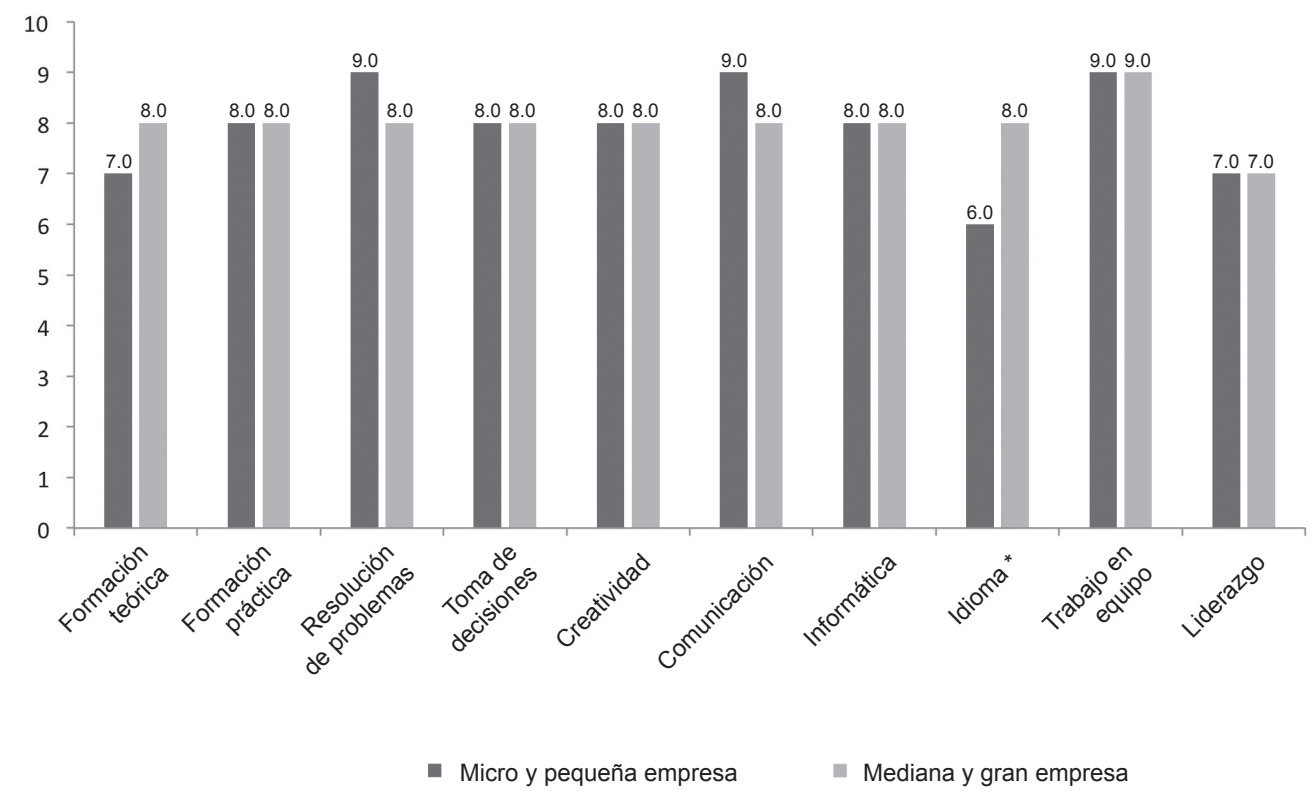

Fuente: Elaboración propia.

* Diferencia estadísticamente significativa a un nivel de significación de .05. 
GRÁFICO 3. Satisfacción de la formación recibida y de las competencias desarrolladas por los titulados (medianas), según los empleadores. Diferencias por tamaño de empresa.

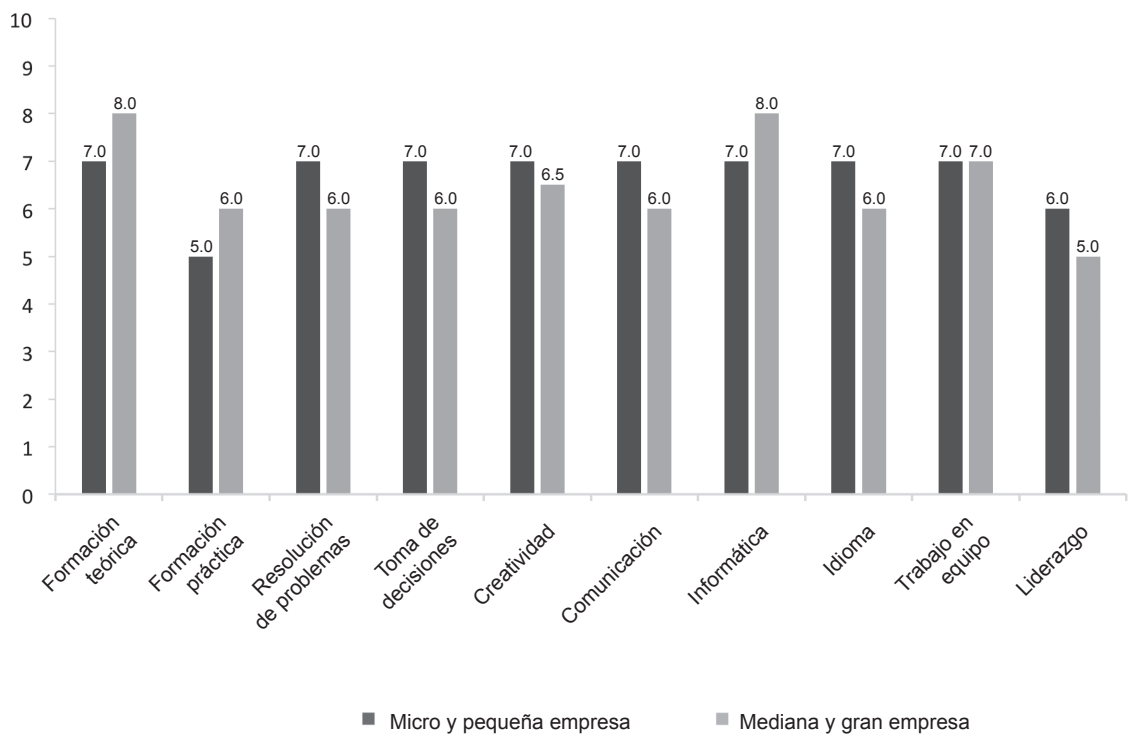

Fuente: Elaboración propia.

* Diferencia estadísticamente significativa a un nivel de significación de .05.

En la encuesta también se preguntaba a los empleadores sobre las dificultades para contratar profesionales de la pedagogía y la psicología adecuados a sus necesidades. Los resultados muestran que el $65 \%$ de los empleadores encuestados han tenido problemas de contratación para ciertos puestos de trabajo; las razones de estos problemas son, para el 33\% de empleadores, que los titulados no tienen las competencias necesarias, y para el 23\%, que faltan titulados en ámbitos concretos. Un $23 \%$ de los empleadores que contestaron a esta pregunta también aportaron otros motivos para la dificultad de cubrir ciertos puestos, como la falta de madurez personal y de motivación, y la falta de conocimiento sobre las funciones del puesto concreto (por ejemplo, se men- ciona el caso de psicólogos y psicopedagogos sin competencias sobre la gestión de la formación y los recursos humanos de la empresa).

6.3. Diferencias en la percepción de las competencias de los titulados, según empleadores y titulados

Para responder al último objetivo (determinar las diferencias entre empleadores y titulados en la valoración de las competencias), se contrastaron las percepciones de los titulados y empleadores a través de la prueba no paramétrica de Mann-Whitney. Los resultados que se pueden observar en el Gráfico 4 indican que existen diferencias estadísticamente significativas en el grado de im- 
portancia que se le otorga a la formación teórica, práctica, creatividad, informática e idioma. Los empleadores valoran más las competencias antes mencionadas (rangos promedio de 259.56, 254.09, 219.93, 238.48, 245.01) en comparación con los titulados (rangos promedio de $178.1,178.9,182.25,181.19,180.23 ; \mathrm{U}=$ 4461, 4723.5, 6113.5, 5473, 5159.5, 7648; $\mathrm{p}<.05)$.
Contrariamente, la competencia de toma de decisiones está valorada como más importante por los titulados (rango promedio de 192.31) que por los empleadores (rango promedio de 158.66; $\mathrm{U}=6439.5 ; p=.038$ ).

Se calculó la $r$ de Rosenthal (1991) para todas las diferencias emergidas significativas, siendo el tamaño del efecto pequeño en todas las competencias (valores $r$ de $.25, .23, .11, .12, .17, .20$ ).

GRÁFICO 4. Diferencias en la importancia de las competencias entre empleadores y titulados (medianas).

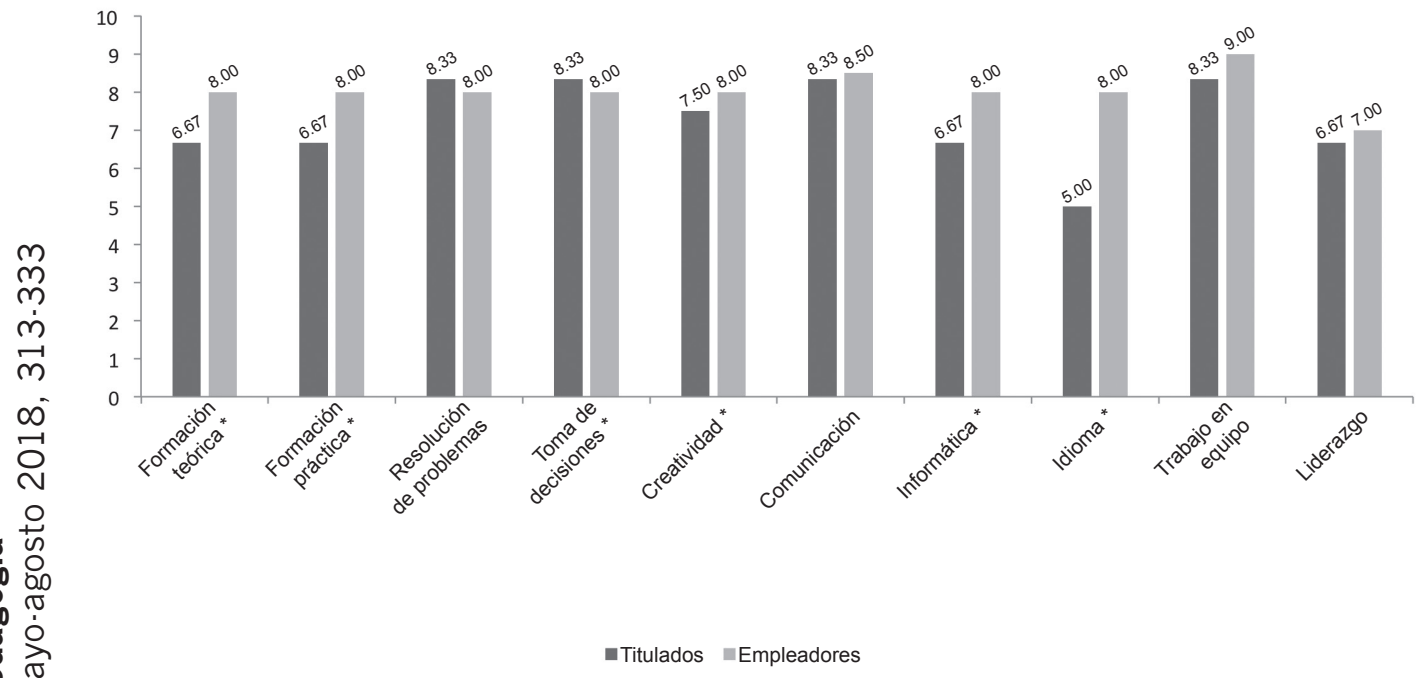

Fuente: Elaboración propia.

* Diferencia estadísticamente significativa a un nivel de significación de .05.

El único grupo de competencias que no presenta diferencias significativas a un nivel de significación de .05 es el de competencias interpersonales, es decir, titulados y empleadores valoran de una forma similar la importancia de estas competencias.
En el Gráfico 5 se muestran las medianas de las puntuaciones del nivel de satisfacción acerca del nivel competencial, con las valoraciones de los titulados y los empleadores. 
GRÁFICo 5. Diferencias entre empleadores y titulados de la satisfacción del nivel de las competencias (medianas).

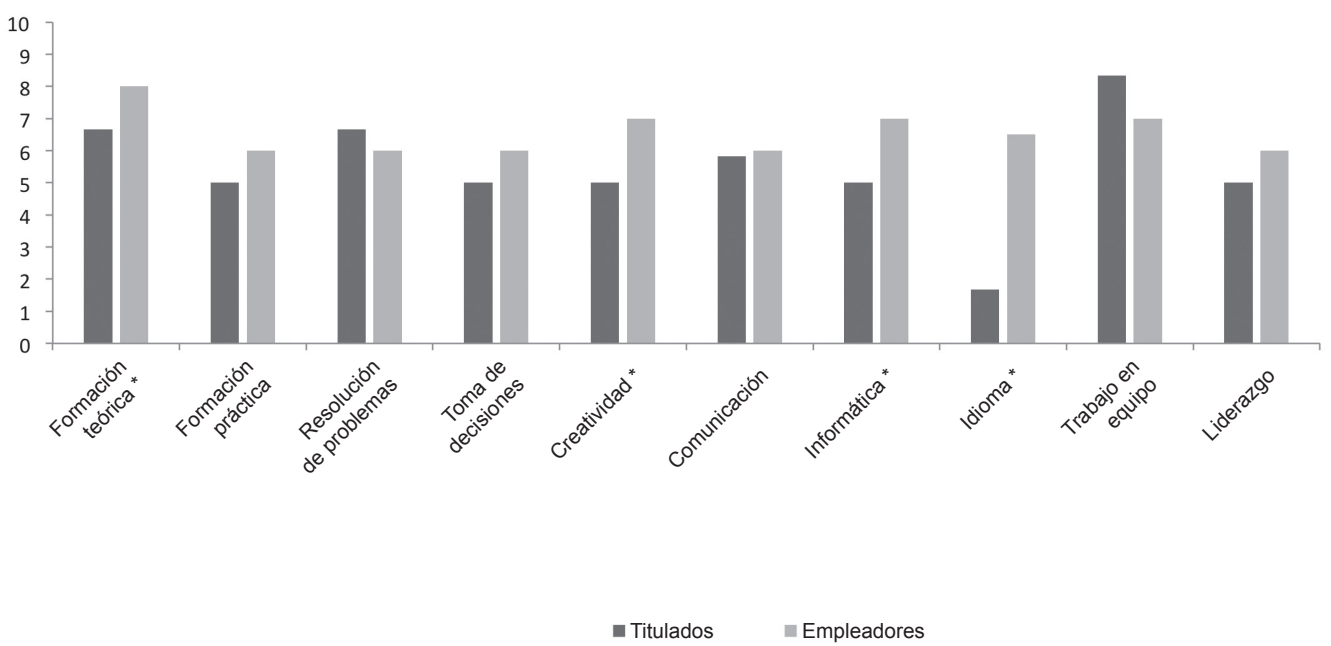

Fuente: Elaboración propia.

* Diferencia estadísticamente significativa a un nivel de significación de .05.

Nuevamente, el grupo de competencias interpersonales no muestra diferencias significativas a un nivel de significación de .05. Solo hay diferencias significativas en el grado de satisfacción con el nivel de formación teórica y en las competencias de creatividad, informática e idioma. En todos los casos, los empleadores presentan un nivel medio de satisfacción mayor (rangos promedio de 217.77, 247.21, 269.67 y 298.24 respectivamente) que los titulados (rangos promedio de 182.78, 178.74, 175.66, 170.28; U=5995.5, 4670.5, $3660,2211.5 ; p<.05)$. Las magnitudes de las diferencias observadas en la satisfacción acerca de la formación teórica, la creatividad y la competencia informática se pueden considerar pequeñas (valores $r$ de $.11, .21$ y .29 respectivamente). En cambio, la diferencia en la satisfacción de las competencias en idiomas presenta un tamaño del efecto moderado $(r=.40)$.

\section{Conclusiones y discusión}

La crisis económica ha generado un empeoramiento de la situación de los jóvenes titulados en el mercado laboral, aumentando su desempleo (MECD, 2013), agravando el desequilibrio entre oferta y demanda (Xiaohao y Changjun, 2013) y empeorando sus condiciones laborales de inserción, en cuanto a ajuste educativo, estabilidad contractual y la posibilidad de obtener un salario para poder independizarse (Pineda-Herrero, Agud-Morell y Ciraso, 2016).

Una vía para analizar el problema es el estudio de la empleabilidad de los recién titulados. Coincidimos con Suárez (2016) en que el concepto de empleabilidad tiene una dimensión externa al individuo, relacionada con los factores macroeconómicos, las políticas de empleo, las condiciones de la oferta laboral, los procesos de selección, 
la oferta formativa disponible y los servicios de apoyo; sin embargo, en este artículo se ha querido profundizar en la faceta de la empleabilidad que tiene que ver con la dimensión individual, con las competencias de los titulados, en concreto con las competencias transversales que han sido descritas en la literatura como de las más relevantes para una buena inserción (Carnevale, Gainer y Meltzer, 1989; Stasz, 1998; Bridge, O’Neill y Cromie, 2003; Stevens, 2005; Weller, 2007; García y Pérez, 2008; Wye y Lim, 2009; Hernández-March, Martín del Peso y Leguey, 2009; Coleman, 2011; Cai, 2013; Bernal, Delgado y Donoso, 2014; Lantarón, 2014, entre otros).

Este artículo se ha centrado en los licenciados en Pedagogía, Psicología y Psicopedagogía, comparando las perspectivas de los propios titulados con la de las empresas que han contratado recientemente a titulados en estas licenciaturas, acerca de su satisfacción con las competencias transversales y su relevancia para el trabajo.

Los resultados del estudio muestran que los titulados otorgan gran importancia a las competencias de trabajo en equipo, resolución de problemas, toma de decisiones y comunicación; estando también mediamente satisfechos con el aprendizaje que han podido realizar en estas competencias, durante la carrera. Están menos satisfechos con la formación que han recibido en idiomas, aunque por lo general no se considera como una competencia muy relevante para el empleo por los titulados de la muestra.

Por su parte, el análisis de las respuestas de los empleadores revela que es- tos consideran como muy importantes las competencias de trabajo en equipo, resolución de problemas, comunicación y creatividad; y están en general satisfechos con el nivel de los titulados contratados, relativo a estas mismas competencias.

Analizando la perspectiva comparada de titulados y empleadores, destaca que ambos colectivos coinciden en valorar el trabajo en equipo como una de las competencias más importantes para trabajar en el sector psicoeducativo, confirmando los resultados obtenidos en otros estudios a nivel internacional (Andrews y Higson, 2010; MetLife, 2011). Respecto a otras competencias, la valoración de titulados y empleadores es significativamente diferente. Especialmente, se observan discrepancias en cuanto a la importancia otorgada a la formación teórica, práctica, a la creatividad, la informática y el conocimiento de idiomas: los empleadores valoran estos dominios como más importantes para el trabajo, y por lo tanto, más relevantes en el proceso de selección (coherentemente con García y Pérez, 2008; Cai, 2013, entre otros). Por otro lado, la toma de decisiones es una competencia sobrevalorada por los titulados respecto a la perspectiva de los empleadores, lo que contrasta con otras investigaciones acerca de la formación deseable para los graduados en psicología y pedagogía (Valeeva y Karimova, 2013). Estos resultados sugieren que las expectativas de las empresas acerca de la formación de los jóvenes titulados no siempre coinciden con las prioridades que los mismos jóvenes dan a ciertos ámbitos competenciales, lo que podría generar un desajuste formativo. 
En cuanto a la satisfacción de los dos colectivos con la formación universitaria en competencias transversales, también se detectan algunas discrepancias interesantes. En concreto, los empleadores se muestran más satisfechos que los titulados en los dominios competenciales de creatividad, informática e idiomas, así como con el nivel de formación teórica de los titulados universitarios. Ambos colectivos están muy satisfechos con las competencias de trabajo en equipo de los jóvenes titulados; dato que corrobora los resultados del estudio cualitativo del AQU (2017), que identifica esta competencia como uno de los puntos fuertes en la formación de los titulados, según las empresas en los sectores de servicios educativos y sociales.

Según los resultados globales de la encuesta (AQU, 2014a), los graduados universitarios consideran que los déficits más importantes de formación se encuentran en las competencias más exigidas por el trabajo: idiomas, toma de decisiones, informática, liderazgo y solución de problemas. Sin embargo, los resultados muestran que los empleadores están más satisfechos con el nivel de competencias de los titulados en Pedagogía, Psicopedagogía y Psicología en informática e idiomas que los propios titulados (destacando sobre todo las competencias en idiomas, donde se ha hallado un tamaño del efecto moderado); mientras que la capacidad de toma de decisiones es considerada menos importante por los empleadores que por los titulados. En cuanto al liderazgo, no es vista como una competencia muy importante (entre las consideradas por el cuestionario) ni para los empleadores, ni para los titulados. A pesar de las limitaciones metodológicas del estudio (en concreto, las características de la muestra de empresas), estos resultados podrían sugerir una visión más optimista de los empleadores sobre las competencias de los jóvenes pedagogos, psicólogos y psicopedagogos, respecto a las de otros titulados de la muestra general del estudio.

De los resultados presentados en este estudio, la resolución de problemas emerge como un ámbito competencial que se podría potenciar en la formación universitaria para responder mejor a las expectativas de los futuros empleadores de pedagogos, psicólogos y psicopedagogos; esta competencia es considerada como muy importante tanto por los empleadores como por los titulados, pero los empleadores no están muy satisfechos con el nivel de dominio de la misma por parte de los titulados. La resolución de problemas complejos es una competencia que se considera cada vez más importante en la selección de candidatos por parte de las empresas (NACE, 2016) y, por ello, se debería enfatizar en el currículum de estas titulaciones.

Respecto a las posibles diferencias en la valoración de las competencias según el tamaño de las empresas donde se emplean a jóvenes titulados, se ha encontrado alguna diferencia significativa pero no coinciden en las valoraciones de empleadores y titulados, y no parecen concluyentes (en línea con aportaciones de Husain, Mokhtar, Ahmad y Mustapha, 2010). La única competencia que empleadores y titulados coinciden en considerar como más importante en las empresas medianas y grandes es la competencia en idiomas, lo 
cual podría estar relacionado con una mayor internacionalización de estas organizaciones.

Una de las principales aportaciones de este estudio es que revela cuáles son las competencias a las que deberían dar más importancia los titulados y las universidades, para responder a las expectativas de los empleadores del sector psicoeducativo. Este estudio nos ha permitido exponer características y diferencias sobre la visión de los empleadores y titulados en el sector psicoeducativo, sobre el que no hay muchos estudios.

Se ha evidenciado que, a pesar de que existen discrepancias en la visión de los titulados en comparación a los empleadores, los psicólogos, pedagogos y psicopedagogos de Cataluña tienen buena parte de las competencias requeridas por las empresas. Sin embargo, casi un tercio de las empresas tienen dificultades para encontrar titulados adecuados para los puestos de trabajo que ofertan. Como indican Singh, Thambusamy y Ramly (2014), las empresas hoy requieren de profesionales con un bagaje competencial amplio, que les de flexibilidad para desarrollar diferentes funciones. A la luz de estos resultados, sería conveniente revisar si la última reforma de los planes de estudios responde mejor a las necesidades de las empresas, y si facilita la inserción laboral de los profesionales de la pedagogía y la psicología en los campos que les son propios.

Se podría concluir que los niveles de paro de los titulados pueden ser atribuidos fundamentalmente a factores del entorno macroeconómico del país (Weller, 2007); pero también, según las respuestas de los empleadores acerca de las dificulta- des de contratación encontradas, pueden responder a algunas carencias en la formación específica de estos jóvenes. Serán necesarias investigaciones más exhaustivas en esta línea para profundizar estos resultados.

\section{Referencias bibliográficas}

Álvarez Llorente, G. y Miles Touya, D. (2006). El papel de la empleabilidad en la satisfacción laboral de los trabajadores temporales. Revista Galega de Economía, 15 (2), 163-182. Recuperado de http://www.usc.es/econo/RGE/ Vol15_2/castelan/art9c.pdf

Andrews, J. y Higson, H. (2008). Graduate employability, 'Soft skills' versus 'hard' business knowledge: a European study. Higher Education in Europe, 33 (4), 411-422.

AQU (2014a). Universitat $i$ Treball a Catalunya. Barcelona: Agència per a la Qualitat del Sistema Universitari de Catalunya.

AQU (2014b). Ocupabilitat $i$ competencies dels graduats recents: l'opinió d'empreses i institucions. Barcelona: Agència per a la Qualitat del Sistema Universitari de Catalunya.

AQU (2017). L'estudi d'ocupadors: anàlisi qualitativa. Barcelona: Agència per a la Qualitat del Sistema Universitari de Catalunya.

Beaven, Z. y Wright, R. (2006). Experience! Experience! Experience! Employer attitudes to arts and event management graduate employability. International Journal of Event Management Research, 2 (1), 17-24.

Bernal, A., Delgado, P. y Donoso, M. (2014). Economía del conocimiento, cultura emprendedora y empleabilidad en el ámbito educativo. Una aproximación al caso español. Procedia Social and Behavioral Sciences, 139, 168-174. Bridge, S., O'Neill, K. y Cromie, S. (2003). Understanding enterprise. Entrepreneurship and small business. Basingstoke: Palgrave. 
Cai, Y. (2013). Graduate employability: a conceptual framework for understanding employers' perceptions. Higher education, 65, 457-469.

Carnevale, A. P. y Smith, N. (2013). Workplace basics: the skills employees need and employers want. Human Resource Development International, 16 (5), 491-501.

Carnevale, A. P., Gainer, L. J. y Meltzer, A. S. (1989). Workplace Basic: The Skills Employers Want. Washington, DC: Eric.

Cohen, J. (1988). Statistical Power Analysis for the Behavioral Sciences (2nd ed.). Hillsdale: Lawrence Erlbaum.

Coleman, J. (2011). Study / Work abroad and employability. University Council of Modern Languages. Recuperado de goo.gl/nYfdWf (Consultado el 21/02/2018).

Colman, A. M., Norris, C. E. y Preston, C. C. (1997). Comparing Rating Scales of Different Lengths: Equivalence of Scores From 5-Point and 7-Point Scales. Psychological Reports, 80 (2), 355-362.

Dawes, J. (2008). Do data characteristics change according to the number of scale points used? An experiment using 5-point, 7-point and 10-point scales. International Journal of Market Research, 50 (1), 61-77.

De Jonge, T., Veenhoven, R. y Arends, L. (2014). Homogenizing Responses to Different Survey Questions on the Same Topic: Proposal of a Scale Homogenization Method Using a Reference Distribution. Social Indicators Research, 117 (1), 275-300.

Freire Seoane, M. J. y Teijeiro Alvarez, M. (2010). Competences of graduates as an indicator of external quality assurance in universities. Regional and Sectoral Economic Studies, 10 (3), 1-15.

Fugate, M., Kinicki, A. J. y Ashforth, B. E. (2004). Employability: A psycho-social construct, its dimensions, and applications. Journal of Vocational Behavior, 65 (1), 14-28.
García, J., Sotelino A. y Crespo, J. (2014). Prospects for Social Employment Insertion of Graduates in Pedagogy in the Autonomous Community of Galicia. From the University to the Labour Market. Procedia - Social and Behavioral Sciences, 139, 412-418.

García Manjón, J. V. y Pérez López, M. C. (2008). Espacio Europeo de Educación Superior, competencias profesionales y empleabilidad. $R e$ vista Iberoamericana de Educación, 46 (9), 1-12. Recuperado de https://rieoei.org/RIE/ article/view/1886

García-Aracil, A. y Van Der Velden, R. (2008). Competencies for young European higher education graduates: Labor market mismatches and their payoffs. Higher Education, 55 (2), 219-239.

Hernández-March, J., Martín del Peso, M. y Leguey, S. (2009). Graduates' Skills and Higher Education: The employers' perspective. Tertiary Education and Management, 15 (1), 1-16.

Husain, M. Y., Mokhtar, S. B., Ahmad, A. A. y Mustapha, R. (2010). Importance of employability skills from employers' perspective. Procedia - Social and Behavioral Sciences, 7, 430-438.

Jackling, B. y De Lange, P. (2009). Do Accounting Graduates' Skills Meet The Expectations of Employers? A Matter of Convergence or Divergence. Accounting Education, 18 (4-5), 369385 .

Lantarón, B. (2014). La empleabilidad en la Universidad Española. Journal for Educators, Teachers and Trainers, 5 (2), 272-286.

Marimuthu, M., Arokiasamy, L. y Ismail, M. (2009). Human Capital Development and Its Impact on Firm Performance: Evidence From Developmental Economics. Journal of International Social Research, 2 (8), 265-272.

Martín-González, M., Ondé, D. y Pérez-Esparrels, C. (2015). El impacto de las competencias en la empleabilidad de los titulados universita- 
rios de las universidades valencianas. Investigaciones de Economía de la Educación, 10, 687-707.

Ministerio de Educación, Cultura y Deporte MECD (2013). Datos Básicos del sistema universitario español. Curso 2013-2014. Recuperado de goo. gl/8HxAuW (Consultado el 21/02/18).

Mccowan, T. (2015). Should universities promote employability? Theory and Research in Education, 13 (3), 267-285.

Medir, L. y Montolio, D. (2015). Satisfacción laboral de los graduados catalanes y los determinantes de su valoración de la formación recibida en la universidad. Revista de Educación y Derecho, 12, 24.

MetLife (2011). The MetLife Survey of the American teachers: Preparing students for college and careers. Recuperado de goo.gl/8wn9cf (Consultado el 21/02/18).

NACE (2016). Job outlook 2016. Bethlehem, PA: National Association of Colleges and Employers.

Pineda-Herrero, P., Agud-Morell, I. y Ciraso-Calí, A. (2016). Factores que intervienen en la inserción laboral de los titulados en Educación en tiempos de crisis: un estudio sobre Cataluña. Revista de Educación, 372, 41-165.

Preston, C. C. y Colman, A. M. (2000). Optimal number of response categories in rating scales: reliability, validity, discriminating power, and respondent preferences. Acta Psychologica, 104 (1), 1-15.

Prokou, E. (2008). The Emphasis on Employability and the Changing Role of the University in Europe. Higher Education in Europe, 33 (4), 387-394.

Rosenthal, R. (1991). Meta-analytic procedures for social research (2nd ed.). Newbury Park, CA: Sage.

Saunders, V. y Zuzel, K. (2010). Evaluating employability skills: Employer and student perception. Bioscience Education, 15 (1), 1-15.
Sefertzi, E. (2000). Creativity. Report produced for the EC funded project INNOREGIO: dissemination of innovation and knowledge management techniques. Recuperado de goo.gl/12nHUk (Consultado el 21/02/18).

Singh, P., Thambusamy, R. X. y Ramly, M. A. (2014). Fit or Unfit? Perspectives of Employers and University Instructors of Graduates' Generic Skills. Procedia - Social and Behavioral Sciences, 123, 315-324.

Stasz, C. (1998). Generic skills at work: implications for occupationally oriented education. En W. J. Nijhof y J. N. Streumer, (Eds.), Key qualifications in work and education (pp. 187206). Dordrecht: Springer.

Stevens, B. (2005). What communication skills do employers want? Silicon Valley recruiters respond. Journal of Employment Counseling, $42,2-9$.

Suárez Lantarón, B. (2016). Empleabilidad: análisis del concepto. Revista de Investigación en Educación, 14 (1), 67-84.

Taylor, A. (2005). What employers look for: the skills debate and the fit with youth perceptions. Journal of Education and Work, 18 (2), 201-218.

Valeeva, R. A. y Karimova, L. S. (2014). Research of Future Pedagogue-psychologists' Social Competency and Pedagogical Conditions of its Formation. Procedia - Social and Behavioral Sciences, 131, 40-44.

Weller, J. (2007). La inserción laboral de los jóvenes: características, tensiones y desafíos. Revista de la Cepal, 92, 61-82.

Wickramasinghe, V. y Perera L. (2010). Graduates', university lecturers' and employers' perceptions towards employability skills. Education + Training, 52 (3), 226-244.

Wye, C. K. y Lim, Y. M. (2009). Perception Differential between Employers and Undergraduates on the Importance of Employability Skills. International Education Studies, 2 (1), 95-105. 
Xiaohao, D. y Changjun, Y. (2013). A Study on the Employment of Graduates of Higher Education in the Context of the Financial Crisis. Procedia - Social and Behavioral Sciences, 77, 164-168.

\section{Biografías de las autoras}

Pilar Pineda-Herrero es Doctora en Ciencias de la Educación y Profesora Titular de Pedagogía Laboral en la Facultad de Educación de la Universidad Autónoma de Barcelona. Dirige el grupo de investigación EFI (Eficacia de la Formación), y forma parte del grupo de investigación GIPE (Grupo Interdisciplinar de Políticas Educativas). Sus líneas de trabajo son la educación y el trabajo, la eficacia de la formación continua y la evaluación del impacto de las políticas formativas.

Anna Ciraso-Calí es diplomada en Ciencias de la Educación (Università de- gli Studi di Padova, Italia) y licenciada en Pedagogía por la Universidad Autónoma de Barcelona. Es investigadora autónoma y colabora en proyectos de investigación con la UAB. Miembro del grupo de investigación EFI y del GIPE. Sus principales ámbitos de investigación son la evaluación de la formación, la transferencia de los aprendizajes, la educación y el trabajo, y la formación profesional del sistema educativo.

Mary Armijos-Yambay es licenciada en Economía por la Escuela Superior Politécnica del Litoral (ESPOL) en Guayaquil, Ecuador, y ha sido Asistente de Investigación en el Centro de Investigaciones Económicas de esta escuela. Realizó sus prácticas preprofesionales en el Departamento de Pedagogía de la Universidad Autónoma de Barcelona. Actualmente trabaja en un proyecto de investigación para obtener su título como economista. 


\section{revista española de pedagogía año LXXVI, n 270, mayo-agosto 2018 \\ Spanish Journal of Pedagogy \\ year LXXVI, n. 270, May-August 2018 \\ sumario*}

table of contents **

\section{Estudios}

\section{Studies}

Xavier Úcar

Metáforas de la intervención socioeducativa:

implicaciones pedagógicas para la práctica

Metaphors for socio-educational intervention:

pedagogical implications for practice

Inmaculada Asensio Muñoz, Elvira Carpintero

Molina, Eva Expósito Casas y Esther López Martín ¿Cuánto oro hay entre la arena? Minería de datos con los resultados de España en PISA 2015

How much gold is in the sand? Data mining with

Spain's PISA 2015 results

Francisco José Fernández-Cruz, Ma José

Fernández-Díaz y Jesús Miguel Rodriguez-Mantilla

Diseño y validación de un instrumento

de medida del perfil de formación docente en

tecnologías de la información y comunicación

209
Design and validation of an instrument to measure teacher training profiles in information and communication technologies

\section{Notas}

Notes

Maria Teresa Caro Valverde,

María Isabel de Vicente-Yagüe Jara

y María Teresa Valverde González

Percepción docente sobre costumbres

metodológicas de argumentación informal

en el comentario de texto

Teacher perception of methodological habits

for informal argumentation in text commentary

Ana Rodriguez-Meirinhos y Esther Ciria-Barreiro

Revisión de intervenciones para mejorar

las habilidades pragmáticas en niños y

niñas con problemas de conducta y atención

Review of interventions to improve pragmatic language

skills in children with behaviour and attention problems

\footnotetext{
* Todos los artículos están publicados en inglés en la página web de la revista: www.revistadepedagogia.org.

** All the articles are published in English on the web page of the journal: www.revistadepedagogia.org.
} 
Pilar Pineda-Herrero, Anna Ciraso-Cali

y Mary Armijos-Yambay

Competencias para la empleabilidad

de los titulados en Pedagogía, Psicología

y Psicopedagogía: un estudio comparativo

entre empleadores y titulados

Employability and competences of Pedagogy,

Psychology, and Educational Psychology graduates:

A comparative study of employers and graduates

Isabel Vilafranca Manguán, Raquel Cercós Raichs y Jordi García Farrero

Los «padres» pedagógicos de Europa. Discursos educativos fundacionales para la integración europea, cien años después de la Gran Guerra

The pedagogical founding fathers of Europe: foundational education discourses for European integration, one hundred years after the First World War

Jonathan Ruiz-Jaramillo y Antonio Vargas-Yáñez

La enseñanza de las estructuras en el Grado de Arquitectura. Metodología e innovación docente a través de las TIC

Teaching structures on Architecture degrees.

ICT-based methodology and teaching innovation

\section{Reseñas bibliográficas}

Gargallo López, B. (Coord.) Enseñanza

centrada en el aprendizaje y diseño por

competencias en la Universidad.

Fundamentación, procedimientos y evidencias de aplicación e investigación

(Vicent Gozálvez). Ballester, L. y Colom, A.

Epistemologías de la complejidad y educación

(Carlos Alberto Pabón Meneses). Monarca, H.

y Thoilliez, B. (Coords.) La profesionalización

docente: debates y propuestas (Francisco Esteban

Bara). Balduzzi, E. Narrazione educativa e

generatività del perdono (Mauricio Bicocca).

373

313

\section{Informaciones}

XVI Conferencia Internacional de la Red

Internacional de Filósofos de la Educación 2018

sobre «Educación, diálogo y esperanza»;

ECER 2018 sobre «lnclusión y Exclusión,

¿Recursos para la Investigación Educativa?»;;

XV Congreso Internacional de Organización

335 de Instituciones Educativas (CIOIE) sobre

«Las tendencias nacionales e internacionales

en organización educativa: entre la estabilidad

y el cambio»;; Los ebooks monográficos de la

revista española de pedagogía.

Una visita a la hemeroteca (Gabriel Álvarez

López). Una visita a la red (David Reyero).

\section{Instrucciones para los autores}

Instructions for authors

Solicitud de originales

Call for papers

403

ISSN 0034-9461 - Depósito legal: M. 6.020 - 1958

e-ISSN 2174-0909 Rev. esp. pedagog. (Internet)

INDUSTRIA GRÁFICA ANZOS, S.L. Fuenlabrada - Madrid 


\title{
Employability and competences of Pedagogy, Psychology, and Educational Psychology graduates: a comparative study of employers and graduates
}

\author{
Competencias para la empleabilidad de los titulados \\ en Pedagogía, Psicología y Psicopedagogía: \\ un estudio comparativo entre empleadores y titulados
}

Pilar PINEDA-HERRERO, PhD. Lecturer. Universidad Autónoma de Barcelona (pilar.pineda@uab.es).

Anna CIRASO-CALI. Graduate. Universidad Autónoma de Barcelona (annaciraso@gmail.com).

Mary ARMIJOS-YAMBAY. Graduate. Escuela Superior Politécnica del Litoral (marbarmi@espol.edu.ec).

\section{Abstract:}

The labour market integration of recent graduates can be influenced by several factors, such as the economic situation of the country, the quality of their university education, and the demands of the labour market.

Although the Spanish economy has recently recovered somewhat, it still has high rates of youth unemployment, even among the best educated groups. Therefore, it is important to analyse in greater depth the transversal competences required and offered in the labour market as these can affect the integration and job satisfaction of university graduates. To analyse the differences in the view of graduates in Pedagogy, Psychology, and Educational Psychology and their employers regarding the competences needed for the labour market this article uses data from the AQU's 'Universitat i Treball a Catalunya' survey (2014a and 2014b) of graduates in these subjects from Catalan universities and of their employers.

The results show discrepancies between employers' and graduates' views. On the one hand, graduates regard some competences as more relevant, such as decision-making, while employers give greater importance to others, such as those relating to information technology and language skills, as well as theoretical and practical training. However, both groups agree that teamwork is the most important competence for work, supporting results obtained in other studies. Furthermore, employers are more satisfied than the graduates with their level in the areas of creativity, computer science, and languages. Problem-solving, however, is an area of competence that could be enhanced in university education.

This study's contribution is to provide evidence based on the retrospective assessment

Revision accepted: 2018-02-10.

This is the English version of an article originally printed in Spanish in issue 270 of the revista española de pedagogía. For this reason, the abbreviation EV has been added to the page numbers. Please, cite this article as follows: Pineda-Herrero, P., CirasoCali, A., \& Armijos-Yambay, M. (2018). Competencias para la empleabilidad de los titulados en Pedagogía, Psicología y Psicopedagogía: un estudio comparativo entre empleadores y titulados | Employability and competen-ces of Pedagogy, Psychology, and Educational Psychology graduates: a comparative study of employers and graduates. Revista Española de Pedagogía, 76 (270), 313-333. doi: https://doi.org/10.22550/REP76-2-2018-06 
of recent graduates that can help align university training with employers' expectations.

Keywords: higher education, competences, vocational adjustment, employers, graduates, youth employment, labour market integration.

\section{Resumen:}

La inserción laboral de los jóvenes universitarios se puede ver influenciada por varios factores, como la situación económica del país, la calidad de la formación universitaria 0 las exigencias del mercado laboral.

A pesar de que la economía española se ha ido recuperando, aún presenta cifras altas de desempleo juvenil, incluso entre los más formados. Por ello, es importante profundizar en el análisis de las competencias transversales que son demandadas y ofertadas en el mercado laboral, ya que de esto puede depender la inserción y satisfacción laboral de los graduados universitarios. En este artículo se analizan, a partir de los datos de la encuesta «Universitat i Treball a Catalunya» de AQU (2014a y 2014b) sobre los titulados en Pedagogía, Psicología y Psicopedagogía en las universidades catalanas y sus empleadores, las diferencias en las perspectivas de ambos colectivos respecto a las competencias necesarias para el mercado laboral.

Los resultados muestran que la visión de empleadores y titulados presenta algunas discrepancias. Por un lado, los titulados consideran más importantes algunas competencias como la toma de decisiones. Sin embargo, los empleadores dan mayor importancia a otras, como las que se relacionan con la informática y el conocimiento de idiomas, o la formación teórica y práctica impartida en la universidad. Ambos colectivos coinciden en posicionar el trabajo en equipo como la competencia más importante para el trabajo, confirmando los resultados obtenidos en otros estudios. Por otro lado, los empleadores están más satisfechos que los propios titulados con su nivel en los dominios competenciales de creatividad, informática e idiomas. La resolución de problemas, en cambio, emerge como un ámbito competencial que se podría potenciar en la formación universitaria.

La contribución del estudio radica en aportar evidencias que puedan ayudar a alinear la formación universitaria con las expectativas de los empleadores, a la luz de la valoración retrospectiva de los recién titulados.

Descriptores: educación superior, competencias, ajuste formativo, empleadores, titulados, empleo juvenil, inserción laboral.

\section{Introduction and theoretical framework}

The labour market integration of young people is a matter that at present deserves to be considered at a social, political, and academic level. The economic crisis has had a legacy in Spain that especially affects the country's recent graduates. While it is true that total unemployment has fallen since 2015, current youth unemployment figures are still alarming. In Catalonia, one of the five autonomous regions with the lowest levels of youth unemployment, the unemployment rate among young people is $30.46 \%$ (data from the INE (National 
Statistics Institute), Active Population Survey, 2017).

The employment situation of young people with university qualifications is especially worrying, given their strategic role for the future of the country and the need for a return on the investment made in them, both privately and as a society. The 2014 workplace integration survey by the INE reported that the employment rate of university graduates in 2010 was $75.6 \%$ and their unemployment rate was $19.2 \%$ in 2014 . By branch of knowledge and qualifications, the unemployment rate for graduates in social and legal sciences was $20.4 \%$, higher than the rate for health sciences or architecture and engineering. Of the three qualifications considered in this study, the one with the best employment rate in 2014 was the degree in Educational Psychology (83\%), followed by the degree in Pedagogy (78\%) and finally Psychology (73.36\%). However, it has been shown that the data on the integration of young education professionals (specifically, graduates from the degrees in Pedagogy, Educational Psychology, and Primary School Teaching) conceal problems with overqualification and precarious employment. According to Pineda-Herrero, Agud-Morell, and Ciraso-Calí (2016), in 2014 just $10.9 \%$ of graduates from 2010 had a satisfactory job where they performed duties appropriate to their level of training, with a minimum annual salary that provided them with economic independence.

The role of universities in training young people has changed in the last decade. One of the main objectives of universities at present is to promote employabil- ity (McCowan, 2015). This role has also been encouraged by the employability policies of the European Union, policies that have led to debates about the relationship between university education and the labour market (Prokou, 2008). However, do universities really train their students so that they can then integrate appropriately into the labour market?

In general terms, employability can be understood as the set of characteristics, competences, and skills that enable somebody to find a job more easily (Álvarez \& Miles, 2006). According to Fugate, Kinicki, and Ashforth (2004), employability is a construct with three dimensions: the identity of the course, personal adaptability, and social and human capital. Here we focus on the dimension of human capital. This is the process of developing knowledge, aptitudes, skills, and values that will improve job satisfaction and performance, at the same time as optimising the functioning of the company (Marimuthu, Arokiasamy, \& Ismail, 2009). Accordingly, there is a need for the competences that are required and offered in the labour market to be analysed in greater depth as the integration and job satisfaction of university graduates can depend on them (García-Aracil \& Van der Velden, 2008).

General or transversal competences are common to most professions. These relate to basic knowledge, the ability to analyse and synthesise, organise and plan, solve problems, take decisions, communicate orally and in writing, language skills, computer skills, handling information, critical thinking, teamwork, interpersonal skills, leadership, research 
skills, autonomy, responsibility, motivation, and self-esteem (Carnevale, Gainer, and Meltzer, 1989; Hernández-March, Martín del Peso, \& Leguey, 2009; Bernal, Delgado, \& Donoso, 2014). Authors like Wye and Lim (2009), Coleman (2011), and Cai (2013) emphasise the importance of knowing foreign languages for good integration, while Carnevale and Smith (2013), and Stevens (2005) show that communication skills, such as oral and written communication and active listening, are very highly valued in numerous professions. García and Pérez (2008) note that to attain the level of productivity demanded by companies in this era of technology, computer and on-line skills are vital. Employers also value team work (Weller, 2007), interpersonal skills (Bridge, O'Neill, \& Cromie, 2003), problem solving, and decision making (Lantarón, 2014; Stasz, 1998).

Recent literature contains studies with quantitative and qualitative focuses into young people's employability and their labour market integration. MartínGonzález, Ondé, \& Pérez-Esparrels (2015) used an exploratory factor analysis and a logit model to examine the impact of competences on the employability of graduates from universities in Valencia; they found that a good grade and workplace and international experience during university studies favour employability. Medir and Montolio (2015) studied the factors associated with the labour market integration of graduates from public universities in Catalonia. They used linear regression models for their analysis and showed a positive impact of university training on job satisfaction. Other stud- ies like the one by Jackling and De Lange (2009) use qualitative methods, such as interviews, to analyse the convergence or divergence between the skills acquired by accounting graduates and employers' expectations. Among the main results, they found that employers seek young people with general skills (such as team work, leadership, verbal communication, and interpersonal skills). These are skills that graduates do not feel that they were taught during their university studies. Consequently, they encountered a discrepancy. There are also mixed-methodology studies, such as the work by Wickramasinghe and Perera (2010) who used data collected from surveys to perform parametric tests to analyse the differences in the employers', university lecturers', and graduates' opinions about the skills needed and acquired for working. Their results indicate that the three groups regard problem-solving, self-confidence, and team work as the most important skills.

There are also empirical studies that analyse young people's competences and employability from the perspective of graduates (for example, García, Sotelino, $\&$ Crespo, 2014) or the perspective of employers (among others, Beaven and Wright, 2006). However, according to Freire and Teijeiro (2010), the approach that can generate the most useful results is to compare the perspectives of both groups, like the one Taylor (2005) used in her evaluation of employers' expectations and how they match the perception of young people, or Saunders and Zuzel (2015) in a study of the convergence of perspectives regarding personal 
qualities, transversal skills, and specific knowledge.

This study will focus on analysing the perceptions of both agents (recent graduates in Pedagogy, Psychology, and Educational Psychology, and employers) regarding the graduates' competences, the importance of these competences in their hiring, and their satisfaction with the training they have received. Its aim is to offer proof of possible mismatches and suggest areas for improvement in university training so that new professionals in education and psychology are more skilled in the job market.

\section{Method}

In this study we set the following objectives:

1) To analyse the perception of graduate competences (importance for work and satisfaction with the training received) from the perspective of the graduates themselves.

2) To analyse the perception of graduate competences (importance for work and satisfaction with the training received) from the perspective of employers.

3) To determine the differences between employers and graduates in their valuation of competences (importance for work and satisfaction with the training received).

\section{Sample}

This study uses the database of the Agència per a la Qualitat del Sistema Universitari de Catalunya (Agency for the Quality of the University System of Catalonia - AQU) regarding the workplace integration of graduates from Catalan universities from the perspective of graduates (AQU, 2014a) and employers (AQU, 2014b).

The sample of graduates in the study includes graduates from all of the public and private universities in Catalonia who graduated in the 2009-2010 academic year. They were surveyed in 2014. It comprises 17,337 people, representing $55 \%$ of the reference population (the people who graduated from Catalan universities in 2010 ) and has a sampling error of $0.51 \%$. For this article, people with degrees in Pedagogy, Psychology, and Educational Psychology who, after graduating, found a job related to their training, were taken into account. After applying these criteria, the sample comprised 328 graduates: 207 with degrees in Psychology, 75 in Pedagogy, and 46 in Educational Psychology.

In the case of employers, organisations that employ educators, psychologists, and educational psychologists were considered. The sample of employers comprises 48 cases.

Table 1 provides a summary of the characteristics of the sample. 
TABLE 1. Description of graduates and employers.

\begin{tabular}{|c|c|c|c|}
\hline & \multirow{3}{*}{\begin{tabular}{|c|} 
Graduates \\
$87 \%$ \\
$10 \%$
\end{tabular}} & \multirow{3}{*}{ Employers } \\
\hline & & & \\
\hline $\begin{array}{l}\text { Current employ- } \\
\text { ment situation }\end{array}$ & $\begin{array}{l}\text { - Employed } \\
\text { - Unemployed }\end{array}$ & & \\
\hline $\begin{array}{l}\text { Area of } \\
\text { employment }\end{array}$ & $\begin{array}{l}\text { - Private organisation } \\
\text { - Public organisation }\end{array}$ & $\begin{array}{l}74 \% \\
26 \% \\
\end{array}$ & $\begin{array}{l}88 \% \\
12 \% \\
\end{array}$ \\
\hline $\begin{array}{l}\text { Main } \\
\text { activity of the } \\
\text { organisation }\end{array}$ & $\begin{array}{l}\text { - Health and social care } \\
\text { - Education, research, and cultural } \\
\text { services }\end{array}$ & $\begin{array}{l}44.2 \% \\
33.1 \%\end{array}$ & $\begin{array}{l}10 \% \\
44 \%\end{array}$ \\
\hline $\begin{array}{l}\text { Size of the } \\
\text { organisation }\end{array}$ & $\begin{array}{l}\text { - Micro organisation (<9 workers) } \\
\text { - Small organisation (10-50 workers) } \\
\text { - Medium organisation (51-250 workers) } \\
\text { - Large organisation (>250 workers) }\end{array}$ & $\begin{array}{l}30 \% \\
27 \% \\
19 \% \\
22 \%\end{array}$ & $\begin{array}{l}21 \% \\
15 \% \\
46 \% \\
19 \% \\
\end{array}$ \\
\hline \multicolumn{2}{|c|}{ Number of recently graduated employees } & 1,298 & \\
\hline
\end{tabular}

Source: Own elaboration.

\section{Instruments and variables}

The study uses the questionnaires from the AQU graduate survey (AQU, 2014b). In the case of the employers, only the questionnaires for companies are used for this article (omitting the questionnaires for educational centres and health centres as the former mainly employ Teaching graduates and the latter Medicine and Nursing).

To be able to choose the data for comparison between employers and graduates regarding competences, specific questions were selected from each questionnaire. In the case of the questionnaire administered to graduates, the «valuation of training received and its appropriateness for work» was selected. In this they were asked to give a valuation ranging from 1 (very low) to 7 (very good) for 14 competences relating to the level of training received in the university and its usefulness for work.
With the employer questionnaire, the responses to Block 3 («competences») were used. These involve evaluating the importance of a list of 15 competences for professional performance and the employer's satisfaction with the recent graduates' training in these competences on a scale of 0 to 10 .

The usefulness of the competences for work (evaluated in the graduate questionnaire) was compared with the level of importance for work (evaluated in the employer questionnaire). Similarly, the valuation of the level of training received (from the graduate questionnaire) was compared with the degree of satisfaction with the training of the graduates (from the employer questionnaire). The competences that were common to the various blocks and questionnaires were chosen: in other words, the ones that could be compared directly. 
In the case of the graduates, a new variable was created with the mean of the competences in oral expression and written communication to create a single variable, called «communication». In the case of the employers, the "creation of new ideas and solutions» variable was treated as being equivalent to the «creativity» variable based on the definition of creativity provided by Sefertzi (2000).

Finally, two blocks of 10 items were used (importance for work and satisfaction with training), administered to graduates and employers alike. Both scales show good reliability $(\alpha=.836$ and $\alpha=.886$ in the graduate sample, $\alpha=.770$ and $\alpha=.886$ in the employer sample). The 10 items on each scale provide information about the following competences, grouped in accordance with the categorisation used by the AQU (2014):

- Theoretical-practical training: theory and practice.

- Cognitive competences: problem solving, decision making, and creativity.

- Instrumental competences: communication, IT, and language.

- Interpersonal competences: team work and leadership.

In addition, items 14 and 15 of Block 2 of the employer questionnaire («hiring processes») were analysed to consider in greater depth the factors that affect the process of hiring of recent graduates. Question 13, which is dichotomous, concerns whether there are difficulties hiring suitable people for a post; if the answer is affirmative, a list of reasons for this problem was suggested, and more than one reason could be chosen.

\section{Procedure}

This study uses a relational-comparative research design with a quantitative focus.

After preliminary descriptive and exploratory analyses, we analysed the valuation of the training received and the competences at an overall level and by size of company using the Mann-Whitney non-parametric $U$ test for rank sums to respond to the first and second objectives. This test was applied due to the type of variable (ordinal) and to the non-compliance with the assumption of normality (assumption evaluated with the Kolmogorov-Smirnov test and explored using normal Q-Q plots and detrended $Q-Q$ plots). For the third objective, we examined whether the perspectives of employers and graduates concerning the importance and satisfaction with the level of competences matched, again using the Mann-Whitney $U$ test. In all cases where this test showed a significant difference, Rosenthal's $r$ (1991) was calculated as a measure of the effect size. As there were no data about the effects of similar variables in previous comparable studies, the magnitude of the $r$ was interpreted following Cohen's statements.

Given that the survey of graduates and the survey of employers use different scales to measure competences, the scales were modified so that the data could be standardised and the competences compared.

To unify data, several methods are recommended such as the linear stretch method, the reference distribution meth- 
od (De Jonge, Veenhoven, \& Arends, 2014), regressions to estimate the new scale (Colman, Norris, \& Preston, 1997), and the formula proposed by Preston and Colman (2000), which is the one used in this study. This formula is: (score-1) / (number of answer categories-1)*100. To make the results easier to interpret, the formula was used following Dawes (2008), multiplying by 10 instead of 100 . This meant that only the graduate scale had to be modified, as the employer one was already from 0 to 10 .

\section{Results}

The results obtained are presented below, arranged by the objectives of the study.

\subsection{Analysis of the view of the compe- tences from the students' perspective \\ To answer the first objective of the study (to analyse the perception of gradu- ates' competences from the perspective of the graduates themselves), we performed a descriptive analysis of the general valu- ation of the training received and compe- tences developed.}

Table 2 shows that the graduates regard team work, problem solving, decision making, and communication competences as very important. Their valuation of these competences regarding the university training they received shows scores that are below the halfway point on the scale (from 0 to 10), although it is necessary to consider the negative asymmetry of all of the distributions, above all the valuation of team work, something that suggests the existence of negative scores that are further from the mean.

The graduates show greater satisfaction with the theory training they received at university, with the attainment of team working, communication, problem solving, and decision-making competences. However, the mean satisfaction with the training received is not high in general, with their valuations of language training standing out as especially negative. It is also apparent that the graduates in the sample do not regard language competences as especially relevant to their work. Nonetheless, the spread of the responses should be noted, with higher standard deviations standing out in the valuations of creativity, IT competences, and languages.

TABLE 2. Description of the graduates' valuation of their competences (importance for work and satisfaction with training).

\begin{tabular}{|c|c|c|c|c|c|c|}
\hline & & Mean & Median & $\begin{array}{l}\text { Standard } \\
\text { deviation }\end{array}$ & Symmetry & Kurtosis \\
\hline \multicolumn{7}{|c|}{ Training and competences: importance for work } \\
\hline \multirow{2}{*}{$\begin{array}{l}\text { Theoretical- } \\
\text { practical } \\
\text { training }\end{array}$} & Theory & 5.737 & 6.670 & 2.461 & -0.397 & -0.212 \\
\hline & Practice & 5.543 & 6.670 & 3.144 & -0.277 & -1.003 \\
\hline
\end{tabular}


Employability and competences of Pedagogy, Psychology, and Educational ...

\begin{tabular}{|c|c|c|c|c|c|c|}
\hline & & Mean & Median & $\begin{array}{l}\text { Standard } \\
\text { deviation }\end{array}$ & Symmetry & Kurtosis \\
\hline \multicolumn{7}{|c|}{ Training and competences: importance for work } \\
\hline \multirow{3}{*}{$\begin{array}{l}\text { Cognitive } \\
\text { competences }\end{array}$} & $\begin{array}{l}\text { Problem } \\
\text { solving }\end{array}$ & 8.130 & 8.330 & 2.194 & -1.447 & 2.029 \\
\hline & $\begin{array}{l}\text { Decision } \\
\text { making }\end{array}$ & 7.884 & 8.330 & 2.310 & -1.282 & 1.353 \\
\hline & Creativity & 6.885 & 7.500 & 2.812 & -0.914 & 0.081 \\
\hline \multirow{3}{*}{$\begin{array}{l}\text { Instrumental } \\
\text { competences }\end{array}$} & Communication & 7.642 & 8.330 & 2.175 & -1.092 & 0.816 \\
\hline & It & 6.392 & 6.670 & 2.904 & -0.614 & -0.462 \\
\hline & Language & 4.969 & 5.000 & 3.500 & -0.069 & -1.298 \\
\hline \multirow{2}{*}{$\begin{array}{l}\text { Interpersonal } \\
\text { competences }\end{array}$} & Team work & 8.226 & 8.330 & 2.064 & -1.189 & 0.979 \\
\hline & Leadership & 6.570 & 6.670 & 2.557 & -0.639 & -0.192 \\
\hline \multicolumn{7}{|c|}{ Training and competences: satisfaction with training received } \\
\hline \multirow{2}{*}{$\begin{array}{l}\text { Theoretical } \\
\text {-practical } \\
\text { training }\end{array}$} & Theory & 6.591 & 6.670 & 2.061 & -0.519 & 0.173 \\
\hline & Practice & 5.081 & 5.000 & 2.848 & -0.069 & -0.811 \\
\hline \multirow{3}{*}{$\begin{array}{l}\text { Cognitive } \\
\text { competencies }\end{array}$} & $\begin{array}{l}\text { Problem } \\
\text { solving }\end{array}$ & 5.843 & 6.670 & 2.503 & -0.459 & -0.450 \\
\hline & $\begin{array}{l}\text { Decision } \\
\text { making }\end{array}$ & 5.571 & 5.000 & 2.632 & -0.220 & -0.771 \\
\hline & Creativity & 4.700 & 5.000 & 2.888 & -0.008 & -0.978 \\
\hline \multirow{3}{*}{$\begin{array}{l}\text { Instrumental } \\
\text { competences }\end{array}$} & Communication & 5.889 & 5.835 & 2.425 & -0.343 & -0.488 \\
\hline & It & 4.518 & 5.000 & 2.858 & 0.069 & -0.950 \\
\hline & Language & 2.434 & 1.670 & 2.684 & 0.866 & -0.248 \\
\hline \multirow{2}{*}{$\begin{array}{l}\text { Interpersonal } \\
\text { competences }\end{array}$} & Team work & 7.276 & 8.330 & 2.309 & -0.698 & -0.067 \\
\hline & Leadership & 4.640 & 5.000 & 2.671 & -0.020 & -0.786 \\
\hline
\end{tabular}

Source: Own elaboration.

To examine these results in depth, the graduates' valuations were analysed to look for possible differences depending on the size of the organisations where they work.

Graph 1 shows the comparison of the graduates' valuation of the usefulness of the training they received, according to the size of the organisations where they work (micro and small organisations; medium and large organisations). The Mann-Whitney U test indicates that computer, language, and team work competences are regarded as most important by recent graduates who work in medium and large organisations (with mean ranks of $176.47,180.32$, and 171.88 respectively), compared with those who work in micro and small organisations 
(mean ranks of 148.22, 145.47, and petences, can be regarded as small ac151.51 respectively), $\mathrm{U}=10,178.5,9,667$, $10,789.5$ with a significance level of $p<.05$. The effect size, in the three comcording to Rosenthal (1991) and Cohen (1988), with $r$ values of .15, .19, and .12 respectively).

GRAPH 1. Valuation of the usefulness of the training received and of the competences for work (median), according to the graduates.

Differences by size of organisation where they work.

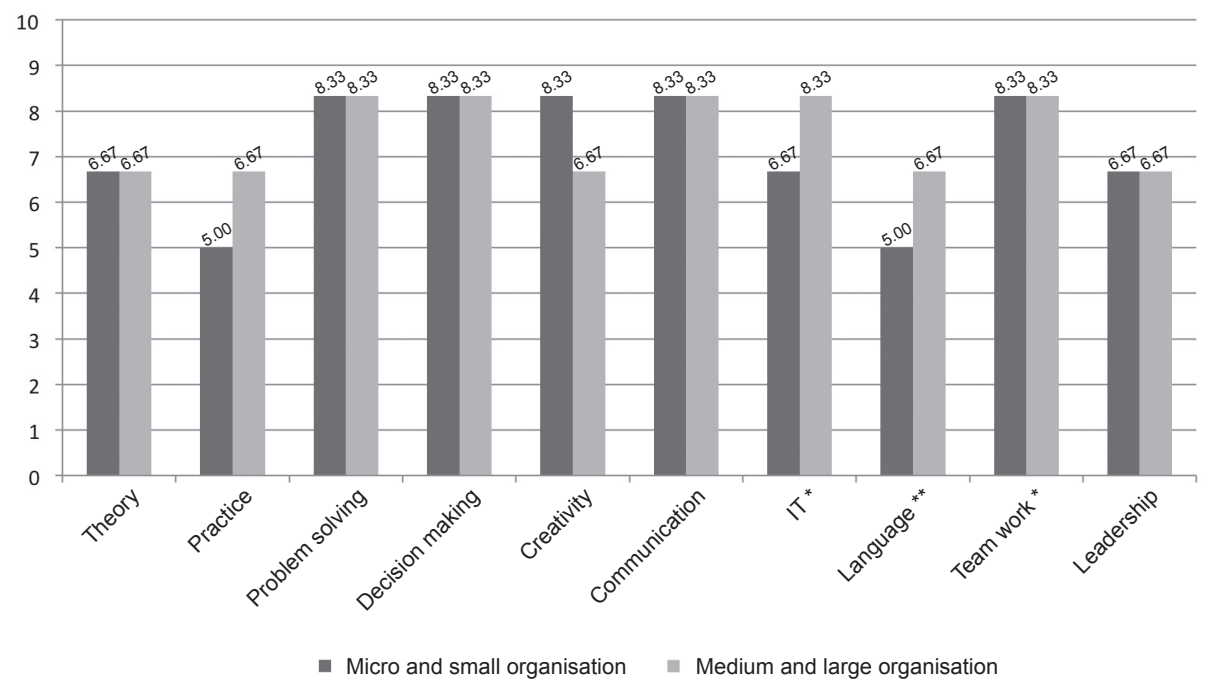

Source: Own elaboration.

* Statistically significant difference at a .05 significance level ** Significance level of .01.

In the block for the level of satisfaction with the training received, the $\mathrm{U}$ test indicates that the graduates who work in medium and large organisations score their satisfaction with training in creativity more highly (with a mean rank of 172.62) than those who work in micro and small organisations (mean rank of 150.97 , $\mathrm{U}=10.690, p=.036)$. The effect size is small $(r=.12)$. The other competences do not present appreciable differences by size at a significance level of .05 .

\subsection{Analysis of competences from the employers' perspective}

Moving on to the second objective of the study (to analyse the perception of the graduates' competences from the employers' perspective), Table 3 shows the descriptive analysis of the general valuation by employers in the sample of the training received by the hired graduates.

It can be seen that employers regard team work, problem solving, communication, and creativity skills as important, these skills being the most highly valued 
ones on average in relation to the com- (which is also the competence they regard petence level attained by the hired grad- as least important for work); however, it uates. The competence with which the is again necessary to note the high level employers are least satisfied is leadership of variance in the responses.

TABLE 3. Description of the valuation of the competences (importance for work and satisfaction with training) by employers.

\begin{tabular}{|c|c|c|c|c|c|c|}
\hline & \\
\hline & & Mean & Median & $\begin{array}{l}\text { Standard } \\
\text { deviation }\end{array}$ & Symmetry & Kurtosis \\
\hline \multicolumn{7}{|c|}{ Training and competences: importance for work } \\
\hline \multirow{2}{*}{$\begin{array}{l}\text { Theoretical } \\
\text {-practical } \\
\text { training }\end{array}$} & Theory & 7.375 & 8.000 & 1.817 & -1.432 & 4.586 \\
\hline & Practice & 7.750 & 8.000 & 2.119 & -1.253 & 2.448 \\
\hline \multirow{3}{*}{$\begin{array}{l}\text { Cognitive } \\
\text { competences }\end{array}$} & $\begin{array}{l}\text { Problem } \\
\text { solving }\end{array}$ & 8.128 & 8.000 & 1.752 & -0.912 & 0.385 \\
\hline & $\begin{array}{l}\text { Decision } \\
\text { making } \\
\end{array}$ & 7.667 & 8.000 & 1.629 & -0.450 & -0.351 \\
\hline & Creativity & 8.043 & 8.000 & 1.517 & -0.465 & -0.417 \\
\hline \multirow{3}{*}{$\begin{array}{l}\text { Instrumental } \\
\text { competences }\end{array}$} & Communication & 8.354 & 8.500 & 1.537 & -1.071 & 1.762 \\
\hline & It & 8.021 & 8.000 & 1.657 & -0.738 & 0.026 \\
\hline & Language & 7.125 & 8.000 & 2.455 & -0.817 & 0.186 \\
\hline \multirow{2}{*}{$\begin{array}{l}\text { Interpersonal } \\
\text { competences }\end{array}$} & Team work & 8.521 & 9.000 & 1.598 & -1.019 & 1.251 \\
\hline & Leadership & 6.674 & 7.000 & 2.066 & -0.520 & 0.546 \\
\hline \multicolumn{7}{|c|}{ Training and competences: level/satisfaction } \\
\hline \multirow{2}{*}{$\begin{array}{l}\text { Theoretical } \\
\text {-practical } \\
\text { training }\end{array}$} & Theory & 7.244 & 8.000 & 1.861 & -1.438 & 3.986 \\
\hline & Practice & 5.511 & 6.000 & 2.212 & -0.319 & -0.012 \\
\hline \multirow{3}{*}{$\begin{array}{l}\text { Cognitive } \\
\text { competences }\end{array}$} & \begin{tabular}{|l|} 
Problem \\
solving
\end{tabular} & 6.273 & 6.000 & 1.744 & 0.221 & 0.317 \\
\hline & \begin{tabular}{|l|} 
Decision \\
making
\end{tabular} & 5.978 & 6.000 & 1.832 & 0.192 & 0.414 \\
\hline & Creativity & 6.444 & 7.000 & 1.791 & -0.265 & 0.915 \\
\hline \multirow{3}{*}{$\begin{array}{l}\text { Instrumental } \\
\text { competences }\end{array}$} & Communication & 6.622 & 6.000 & 2.037 & -0.455 & 1.346 \\
\hline & It & 7.044 & 7.000 & 2.163 & -0.765 & 0.521 \\
\hline & Language & 6.068 & 6.500 & 2.204 & -0.241 & -0.413 \\
\hline \multirow{2}{*}{$\begin{array}{l}\text { Interpersonal } \\
\text { competences }\end{array}$} & Team work & 7.000 & 7.000 & 1.638 & -0.227 & 0.471 \\
\hline & Leadership & 5.273 & 6.000 & 2.316 & -0.042 & 0.087 \\
\hline
\end{tabular}

Source: Own elaboration. 
GRAPH 2. Valuation of the importance of training received and of the competences for work (median) according to employers. Differences by size of company.

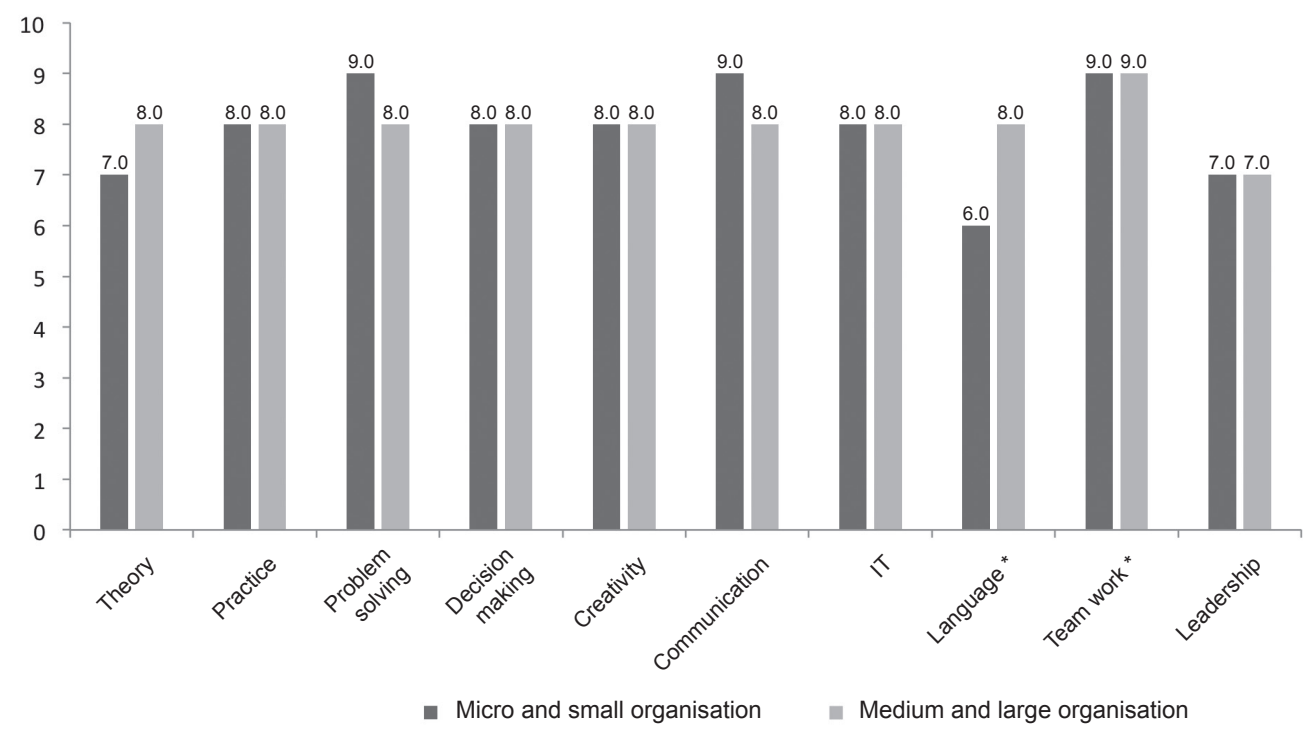

Source: Own elaboration.

* Statistically significant difference at a .05 significance level.

$\stackrel{m}{m}$

$m$

$m$

$\vec{m}$

$\infty$

궁

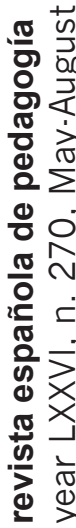

Source: Own elaboration

* Statistically significant difference at a .05 significance level.
GRAPH 3. Satisfaction with the importance of the training received and the competences developed by the graduates (median), according to employers. Differences by size of company.

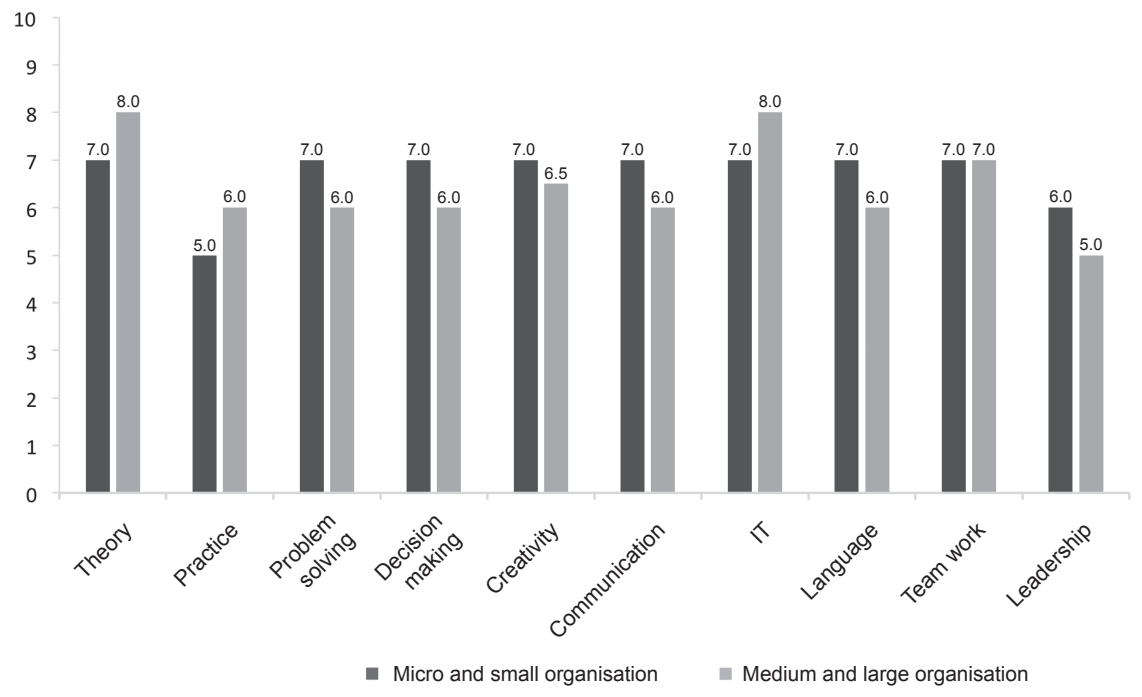

Medium and large organisation 
As in the previous section, these results have been analysed to seek possible differences between the valuation of competences by size of organisation (micro and small organisations; medium and large organisations) using the Mann-Whitney U test. As shown in Graphs 2 and 3, the only difference with statistical significance at $p<.05$ is in the valuation of language competences: employers from medium and large companies regard them as more important (mean rank of 27.89) than employers from micro and small companies (mean rank of $18.32, \mathrm{U}=158.5$, $p=.021$ ). The magnitude of this difference is moderate $(r=.33)$.

The survey also asked employers about difficulties in hiring pedagogy and psychology professionals who meet their needs. The results show that $65 \%$ of the employers surveyed have had problems hiring for certain posts; the reasons for these problems are, for $33 \%$ of employers, that graduates do not have the necessary competences and, for $23 \%$, that there is a lack graduates in specific areas. Other reasons were given by $23 \%$ of the employers who answered this question for the difficulty of covering certain posts, such as lack of personal maturity and motivation, and lack of knowledge about the functions of the specific role (for example, they mention the case of psychologists and educational psychologists who do not have competences relat- ing to the company's training and human resources).

\subsection{Differences in perception of the competences of the graduates, accord- ing to employers and graduates}

To answer the last objective (to establish the differences, between employers and graduates in their valuation of competences), the perceptions of graduates and employers were compared using the Mann-Whitney non-parametric test. The results shown in Graph 4 indicate that there are statistically significant differences in the level of importance given to theory and practical training, creativity, IT, and languages. The employers value more highly the competences mentioned above (mean ranks of 259.56, 254.09, $219.93,238.48,245.01)$ in comparison with the graduates (mean ranks of 178.1, $178.9,182.25,181.19,180.23$; U $=4,461$, 4,723.5, 6,113.5, 5,473, 5,159.5, 7,648; $p$ $<.05)$.

In contrast, the decision-making competence is regarded as more important by graduates (average range of 192.31) than employers (mean rank of 158.66; $\mathrm{U}=6,439.5 ; p=.038$ ).

Rosenthal's $r$ (1991) was calculated for all of the significant differences that emerged. The effect size was small for all of the competences ( $r$ values of $.25, .23$, $.11, .12, .17, .20)$. 
GRAPH 4. Differences in the importance of the competences between employers and graduates (median).

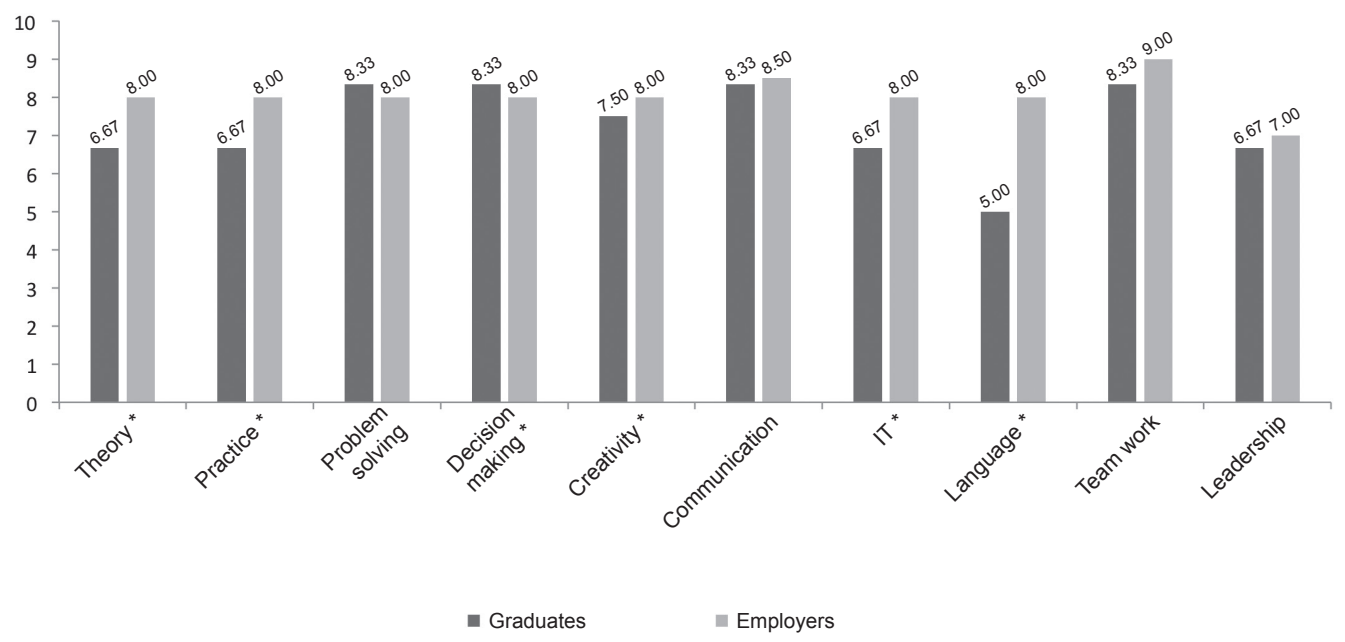

Source: Own elaboration.

* Statistically significant difference at a .05 significance level.

$\infty$
The only group of competences that do not display significant differences at a .05 significance level are interpersonal com- petences. In other words, graduates and employees have a similar valuation of the importance of these competences.

GRAPH 5. Differences between employers and graduates in satisfaction with the level of the competences (median).

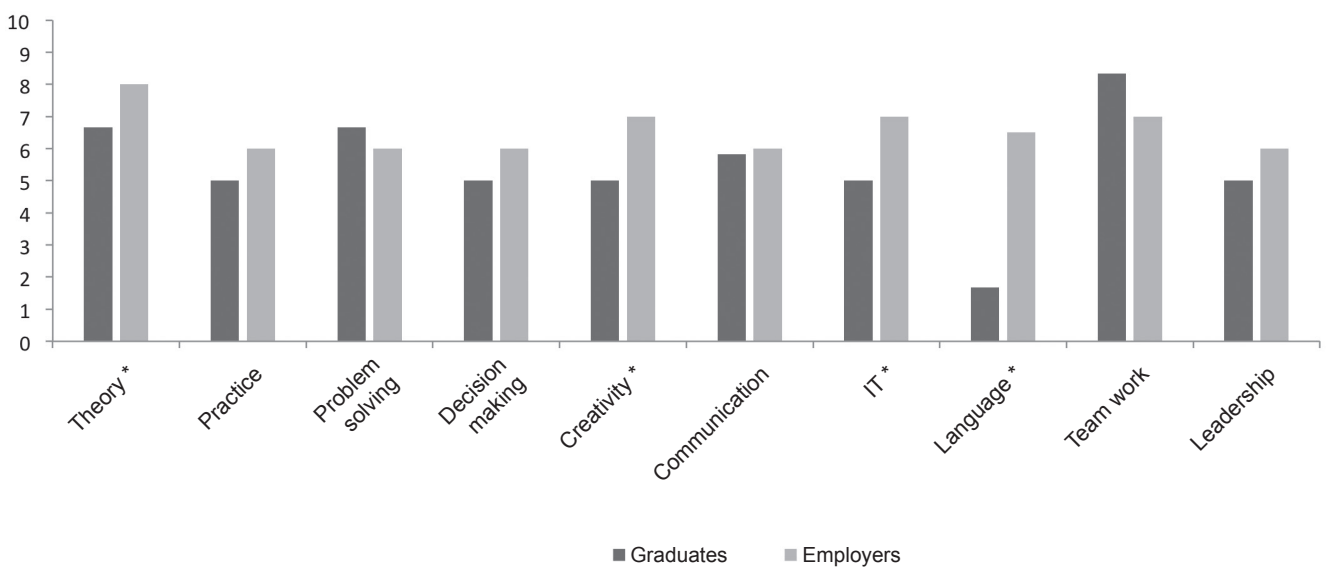

Source: Own elaboration.

* Statistically significant difference at a .05 significance level. 
Graph 5 shows the median values for the scores for the level of satisfaction with the level of competences, with the valuations of the graduates and employers.

Again, the interpersonal competences group does not display significant differences at a significance level of .05. There are only significant differences in the level of satisfaction with training in theory and in the creativity, IT, and language competences. In all cases, the employers have a higher mean level of satisfaction (mean ranks of 217.77, 247.21, 269.67, and 298.24 respectively) than the graduates (mean ranks of 182.78, 178.74, 175.66, 170.28; U=5,995.5, 4,670.5, $3,660,2,211.5 ; p<.05)$. The magnitudes of the differences observed in the satisfaction with theory training, creativity, and IT competences can be regarded as small ( $r$ values of .11, .21, and .29 respectively). In contrast, the difference in the level of satisfaction with language competences displays a moderate effect size $(r=.40)$.

\section{Conclusions and discussion}

The economic crisis has led to a worsening of the situation of recent graduates in the labour market and an increase in their unemployment rate (MECD, 2013), exacerbating the imbalance between supply and demand (Xiaohao and Changjun, 2013) and worsening the conditions of their labour market integration concerning educational alignment, contractual stability, and the possibility of obtaining a large enough salary to be able to be independent (Pineda-Herrero, Agud-Morell, and Ciraso, 2016).
One way to analyse this problem is to study the employability of recent graduates. We agree with Suárez (2016) that the concept of employability has a dimension that is extrinsic to the individual and relates to macroeconomic factors, employment policies, labour supply conditions, selection processes, the range of training available, and support services. Nonetheless, in this article we have attempted to examine in depth the facet of employability that relates to the individual dimension, with the competences of graduates, specifically the transversal competences that have been described in the literature as being most important for good workplace integration (among others: Carnevale, Gainer, \& Meltzer, 1989; Stasz, 1998; Bridge, O’Neill, \& Cromie, 2003; Stevens, 2005; Weller, 2007; García \& Pérez, 2008; Wye \& Lim, 2009; Hernández-March, Martín del Peso, \& Leguey, 2009; Coleman, 2011; Cai, 2013; Bernal, Delgado, and Donoso, 2014; Lantarón, 2014).

This article focuses on Pedagogy, Psychology, and Educational Psychology graduates, comparing the perspectives of graduates themselves and of the business who have recently hired graduates in these subjects regarding their satisfaction with transversal competences and their relevance to work.

The results of the study show that the graduates regard team work, problem solving, decision making, and communication competences as very important. They are also on average satisfied with what they were able to learn in these competences during their degree. They are less satisfied with the training they received 
in languages, although the graduates in the sample do not generally regard this as a particularly relevant competence for employment.

For its part, the analysis of employers' responses shows that they regard team work, problem solving, communication, and creativity competences as very important, and that they are generally satisfied with the hired graduates' level in these competences.

Analysis of the compared perspectives of graduates and employers shows that both groups value team work as one of the most important competences for working in the psycho-educational sector, confirming the results obtained in other studies at the international level (Andrews and Higson, 2010; MetLife, 2011). As for other competences, the valuations by graduates and employers differ significantly. In particular, discrepancies are apparent regarding the importance attributed to, creativity, IT, languages, theoretical and practical training: employers regard these areas as more important for work, and so as having greater weight in the selection process (agreeing with García and Pérez, 2008, Cai, 2013, and others). In contrast, decision making is a competence that graduates overvalue in comparison with the views of the employers, something that contrasts with other research into what training is desirable for graduates in Psychology and Pedagogy (Valeeva \& Karimova, 2013). These results suggest that the employers' expectations about the training of the recent graduates do not always coincide with the priorities that the graduates themselves give to certain areas of competence, some- thing that could create an imbalance in training.

As for the satisfaction of the two groups with university training in transversal competences, some interesting discrepancies were also detected. Specifically, employers report greater satisfaction than graduates with the competence areas of creativity, IT, and languages, as well as with the level of theoretical training of the university graduates. Both groups are very satisfied with the teamwork competences of the recent graduates, a factor that corroborates the results of the AQU's qualitative study (2017) which identifies this competence as one of the strong points in the training of the graduates according to businesses in the educational and social service sectors.

According to the overall results of the survey (AQU, 2014a), university graduates believe that the most important training deficits are in the competences that are most wanted in work: languages, decision making, IT, leadership, and problem solving. However, the results show that employers are more satisfied with the competence level of Pedagogy, Psychology, and Educational Psychology graduates in IT and languages than the graduates themselves are (in particular, language competences stand out, where a moderate effect size was found), while employers regard the ability to make decisions as less important than graduates do. Regarding leadership, neither employers nor graduates regard this competence as particularly important (among those covered by the questionnaire). Despite the methodological limitations of 
the study (specifically, the characteristics of the sample of organisations), these results could suggest that the employers have a more optimistic view of the competences of young educators, psychologists, and educational psychologists compared with other graduates in the general study sample.

From the results presented in this study, problem solving emerges as an area of competence that could be strengthened in university training to better meet future employers' expectations of educators, psychologists, and educational psychologists; this competence is regarded as very important by both employers and graduates, but the employers are not very satisfied with the graduates' level of command of it. Solving complex problems is a competence that is increasingly regarded as important in the selection of candidates by businesses (NACE, 2016) and so it should be emphasised in the curriculums of these qualifications.

Regarding the possible differences in the valuation of the competences according to the size of the organisations that where the recent graduates work, some significant differences were observed, but these do not match the valuations by employers and graduates, and they do not seem to be conclusive (in line with contributions by Husain, Mokhtar, Ahmad, and Mustapha, 2010). The only competence that employers and graduates both regard as more important in medium and large businesses is languages, something that could relate to a higher level of internationalisation in these organisations.
One of this study's main contributions is that it shows which competences should be regarded as more important by graduates and universities to meet the expectations of employers in the psycho-educational sector. This study has permitted us to present the characteristics and differences of views of employers and graduates in the psycho-educational sector, a topic on which there are few studies.

It has shown that, although there are discrepancies between the views of graduates and employers, the psychologists, educators, and educational psychologists of Catalonia have a good part of the transversal competences required by companies. Nonetheless, almost a third of companies have difficulties finding appropriate graduates for the posts they offer. As Singh, Thambusamy, and Ramly indicate (2014), companies today require professionals with a broad selection of competences, giving them the flexibility to perform different functions. In light of these results, it would be helpful to check whether the latest reform to syllabuses better meets the needs of the companies, and whether it facilitates the workplace integration of the pedagogy and psychology professionals in the fields that pertain to them.

It might be concluded that graduate unemployment levels could fundamentally be attributed to factors from the macroeconomic climate of the country (Weller, 2007), but, according to employers' responses concerning the difficulties they have encountered in hiring, these unemployment levels could also be the result of particular shortcomings in the spe- 
cific training of these young people. More exhaustive research along these lines will be needed to explore these results in greater depth.

\section{References}

Álvarez Llorente, G. \& Miles Touya, D. (2006). El papel de la empleabilidad en la satisfacción laboral de los trabajadores temporales. Revista Galega de Economía, 15 (2), 163-182. Retrieved from http://www.usc.es/econo/RGE/ Vol15_2/castelan/art9c.pdf

Andrews, J. \& Higson, H. (2008). Graduate employability, 'Soft skills' versus 'hard' business knowledge: a European study. Higher Education in Europe, 33 (4), 411-422.

AQU (2014a). Universitat $i$ Treball a Catalunya. Barcelona: Agència per a la Qualitat del Sistema Universitari de Catalunya. AQU (2014b). Ocupabilitat $i$ competències dels graduats recents: l'opinió d'empreses i institucions. Barcelona: Agència per a la Qualitat del Sistema Universitari de Catalunya.

AQU (2017). L'estudi d'ocupadors: anàlisi qualitativa. Barcelona: Agència per a la Qualitat del Sistema Universitari de Catalunya.

Beaven, Z. \& Wright, R. (2006) Experience! Experience! Experience! Employer attitudes to arts $\&$ event management graduate employability. International Journal of Event Management Research, 2 (1), 17-24.

Bernal, A., Delgado, P., \& Donoso, M. (2014). Economía del conocimiento, cultura emprendedora y empleabilidad en el ámbito educativo. Una aproximación al caso español. Procedia - Social and Behavioral Sciences, 139, 168-174.

Bridge, S., O'Neill, K., \& Cromie, S. (2003). Understanding enterprise. Entrepreneurship and small business. Basingstoke: Palgrave.
Cai, Y. (2013). Graduate employability: a conceptual framework for understanding employers' perceptions. Higher education, 65, 457-469.

Carnevale, A. P. \& Smith, N. (2013). Workplace basics: the skills employees need and employers want. Human Resource Development International, 16 (5), 491-501.

Carnevale, A. P., Gainer, L. J., \& Meltzer, A. S. (1989). Workplace Basics: The Skills Employers Want. Washington, DC: Eric.

Cohen, J. (1988). Statistical Power Analysis for the Behavioral Sciences (2nd ed.). Hillsdale: Lawrence Erlbaum.

Coleman, J. (2011). Study / Work abroad and employability. University Council of Modern Languages. Retrieved from goo.gl/nYfdWf (Consulted on 21/02/2018).

Colman, A. M., Norris, C. E., \& Preston, C. C. (1997). Comparing Rating Scales of Different Lengths: Equivalence of Scores From 5-Point and 7-Point Scales. Psychological Reports, 80 (2), 355-362.

Dawes, J. (2008). Do data characteristics change according to the number of scale points used? An experiment using 5-point, 7-point and 10-point scales. International Journal of Market Research, 50 (1), 61-77.

De Jonge, T., Veenhoven, R., \& Arends, L. (2014). Homogenizing Responses to Different Survey Questions on the Same Topic: Proposal of a Scale Homogenization Method Using a Reference Distribution. Social Indicators Research, 117 (1), 275-300.

Freire Seoane, M. J. \& Teijeiro Alvarez, M. (2010). Competences of graduates as an indicator of external quality assurance in universities. Regional and Sectoral Economic Studies, 10 (3), 1-15.

Fugate, M., Kinicki, A. J., \& Ashforth, B. E. (2004). Employability: A psycho-social construct, its dimensions, and applications. Journal of Vocational Behavior. 65 (1), 14-28. 
García, J., Sotelino A., \& Crespo, J. (2014). Prospects for Social Employment Insertion of Graduates in Pedagogy in the Autonomous Community of Galicia. From the University to the Labour Market. Procedia - Social and Behavioral Sciences, 139, 412-418.

García Manjón, J. V. \& Pérez López, M. C. (2008). Espacio Europeo de Educación Superior, competencias profesionales y empleabilidad. Revista Iberoamericana de Educación, 46 (9), 1-12. Retrieved from https://rieoei.org/RIE/ article/view/1886

García-Aracil, A. \& Van Der Velden, R. (2008). Competencies for young European higher education graduates: Labor market mismatches and their payoffs. Higher Education, 55 (2), 219-239.

Hernández-March, J., Martín del Peso, M., \& Leguey, S. (2009). Graduates' Skills and Higher Education: The employers' perspective. Tertiary Education and Management, 15 (1), 1-16.

Husain, M. Y., Mokhtar, S. B., Ahmad, A. A., \& Mustapha, R. (2010). Importance of employability skills from employers' perspective. Procedia - Social and Behavioral Sciences, 7, 430-438.

Jackling, B. \& De Lange, P. (2009). Do Accounting Graduates' Skills Meet The Expectations of Employers? A Matter of Convergence or Divergence. Accounting Education, 18 (4-5), 369-385.

Lantarón, B. (2014). La empleabilidad en la Universidad Española. Journal for Educators, Teachers and Trainers, 5 (2), 272-286.

Marimuthu, M., Arokiasamy, L., \& Ismail, M. (2009). Human Capital Development and Its Impact on Firm Performance: Evidence From Developmental Economics. Journal of International Social Research, 2 (8), 265-272.

Martín-González, M., Ondé, D., \& Pérez-Esparrels, C. (2015). El impacto de las competencias en la empleabilidad de los titulados universitarios de las universidades valencianas. Investigaciones de Economía de la Educación, 10, 687-707.

Ministerio de Educación, Cultura y Deporte [MECD]. (2013). Datos Básicos del sistema universitario español. Curso 2013-2014. Madrid: Centro de Publicaciones. Ministerio de Educación, Cultura y Deporte. Retrieved from g0o.gl/8HxAuW (Consulted on 21/02/18).

McCowan, T. (2015). Should universities promote employability? Theory and Research in Education, 13 (3), 267-285.

Medir, L. \& Montolio, D. (2015). Satisfacción laboral de los graduados catalanes y los determinantes de su valoración de la formación recibida en la universidad. Revista de Educación y Derecho, 12, 24.

MetLife (2011). The MetLife Survey of the American teachers: Preparing students for college and careers. Retrieved from goo.gl/8wn $9 \mathrm{cf}$ (Consulted on 21/02/18).

NACE (2016). Job outlook 2016. Bethlehem, PA: National Association of Colleges and Employers.

Pineda-Herrero, P., Agud-Morell, I., \& Ciraso-Calí, A. (2016). Factores que intervienen en la inserción laboral de los titulados en Educación en tiempos de crisis: un estudio sobre Cataluña. Revista de Educación, 372, 41-165.

Preston, C. C. \& Colman, A. M. (2000). Optimal number of response categories in rating scales: reliability, validity, discriminating power, and respondent preferences. Acta Psychologica, 104 (1), 1-15.

Prokou, E. (2008). The Emphasis on Employability and the Changing Role of the University in Europe. Higher Education in Europe, 33 (4), 387-394.

Rosenthal, R. (1991). Meta-analytic procedures for social research (2nd ed.). Newbury Park, CA: Sage. 
Saunders, V. \& Zuzel, K. (2010). Evaluating employability skills: Employer and student perception. Bioscience Education, 15 (1), 1-15.

Sefertzi, E. (2000). Creativity. Report produced for the EC funded project INNOREGIO: dissemination of innovation and knowledge management techniques. Retrieved from goo.gl/12nHUk (Consulted on 21/02/18).

Singh, P., Thambusamy, R. X., \& Ramly, M. A. (2014). Fit or Unfit? Perspectives of Employers and University Instructors of Graduates' Generic Skills. Procedia - Social and Behavioral Sciences, 123, 315-324.

Stasz, C. (1998). Generic skills at work: implications for occupationally oriented education. In W. J. Nijhof \& J. N. Streumer, (Eds.), Key qualifications in work and education (pp. 187206). Dordrecht: Springer.

Stevens, B. (2005). What communication skills do employers want? Silicon Valley recruiters respond. Journal of Employment Counseling, $42,2-9$.

Suárez Lantarón, B. (2016). Empleabilidad: análisis del concepto. Revista de Investigación en Educación, 14 (1), 67-84.

Taylor, A. (2005). What employers look for: the skills debate and the fit with youth perceptions. Journal of Education and Work, 18 (2), 201-218.

Valeeva, R. A. \& Karimova, L. S. (2014). Research of Future Pedagogue-psychologists' Social Competency and Pedagogical Conditions of its Formation. Procedia - Social and Behavioral Sciences, 131, 40-44.

Weller, J. (2007). La inserción laboral de los jóvenes: características, tensiones y desafíos. Revista de la Cepal, 92, 61-82.

Wickramasinghe V. \& Perera L. (2010). Graduates', university lecturers' and employers' perceptions towards employability skills. Education + Training, 52 (3), 226-244.
Wye, C. K. \& Lim, Y. M. (2009). Perception Differential between Employers and Undergraduates on the Importance of Employability Skills. International Education Studies, 2 (1), 95-105.

Xiaohao, D. \& Changjun, Y. (2013). A Study on the Employment of Graduates of Higher Education in the Context of the Financial Crisis. Procedia - Social and Behavioral Sciences, 77, 164-168.

\section{Authors' biographies}

Pilar Pineda-Herrero has a $\mathrm{PhD}$ in Educational Sciences and Lecturer in Occupational Pedagogy in the Faculty of Education of the Universidad Autónoma de Barcelona. She leads the EFI (Efficacy of Training) research group and is a member of the GIPE (Interdisciplinary Educational Policies Group) research group. Her lines of research are education and work, the efficacy of continuous training, and evaluating the impact of training policies.

Anna Ciraso-Calí holds a diploma in Educational Sciences (Università degli Studi di Padova, Italia) and has a degree in Pedagogy from the Universidad Autónoma de Barcelona. She is an independent researcher and cooperates on research projects with the UAB. Her main areas of interest are evaluating training, transfer of learning, education and work, and initial vocational education and training. She is a member of the EFI (Efficacy of Training) research group and of the GIPE (Interdisciplinary Educational Policies Group) research group.

Mary Armijos-Yambay is a graduate of the Escuela Superior Politécnica del Li- 
toral (ESPOL) in Guayaquil, Ecuador and in the Department of Pedagogy of the she was a research assistant in the Eco- Universitat Autònoma de Barcelona. She nomics Research Centre at ESPOL. She is currently working on a research project completed her preprofessional practices to qualify as an economist. 


\section{revista española de pedagogía año LXXVI, n 270, mayo-agosto 2018 \\ Spanish Journal of Pedagogy \\ year LXXVI, n. 270, May-August 2018}

\section{Table of Contents}

\section{Studies}

Xavier Úcar

Metaphors for socio-educational intervention:

pedagogical implications for practice

209

Inmaculada Asensio Muñoz, Elvira Carpintero Molina,

Eva Expósito Casas and Esther López Martín

How much gold is in the sand? Data mining

with Spain's PISA 2015 results

225

Francisco José Fernández-Cruz, Ma José Fernández-

Díaz and Jesús Miguel Rodriguez Mantilla

Design and validation of an instrument

to measure teacher training profiles in

information and communication technologies

\section{Notes}

María Teresa Caro Valverde, María Isabel de VicenteYagüe Jara and Maria Teresa Valverde González Teacher perception of methodological habits for informal argumentation in text commentary
Ana Rodriguez-Meirinhos and Esther Ciria-Barreiro

Review of interventions to improve pragmatic language skills in children with behaviour and attention problems 295

Pilar Pineda-Herrero, Anna Ciraso-Cali and Mary Armijos-Yambay

Employability and competences of Pedagogy,

Psychology, and Educational Psychology

graduates: A comparative study of employers and graduates

Isabel Vilafranca Manguán,

Raquel Cercós Raichs and Jordi García Farrero

The pedagogical founding fathers of Europe:

247 foundational education discourses

for European integration, one hundred

years after the First World War

Jonathan Ruiz-Jaramillo and

Antonio Vargas-Yáñez

Teaching structures on Architecture degrees. ICT.

273 based methodology and teaching innovation 


\section{Book reviews}

Gargallo López, B. (Coord.) Enseñanza centrada

en el aprendizaje y diseño por competencias en la

Universidad. Fundamentación, procedimientos

y evidencias de aplicación e investigación

[Learning.centred teaching and designing for

skills in the university: foundations, procedures,

and evidence for application to research]

(Vicent Gozálvez). Ballester, L. \& Colom, A.

Epistemologías de la complejidad y educación

[Epistemologies of complexity and education]
(Carlos Alberto Pabón Meneses).

Monarca, H. \& Thoilliez, B. (Coords.)

La profesionalización docente: debates

y propuestas [The professionalisation

of teaching: debates and proposals]

(Francisco Esteban Bara).

Balduzzi, E. Narrazione educativa e generatività

del perdono [Educational narration and the

creation of forgiveness] (Mauricio Bicocca).

373

Call for papers

403

This is the English version of the research articles and book reviews published originally in the Spanish printed version of issue 270 of the revista española de pedagogía. The full Spanish version of this issue can also be found on the journal's website http://revistadepedagogia.org.

ISSN: 0034-9461 (Print), 2174-0909 (Online)

https://revistadepedagogia.org/

Depósito legal: M. 6.020 - 1958

INDUSTRIA GRÁFICA ANZOS, S.L. Fuenlabrada - Madrid 